\title{
Temporally semidiscrete approximation of a Dirichlet boundary control for a fractional/normal evolution equation with a final observation *
}

\author{
QIN ZHOU ${ }^{\dagger}$ AND BINJIE Li ${ }^{\ddagger}$
}

School of Mathematics, Sichuan University.

\begin{abstract}
Optimal Dirichlet boundary control for a fractional/normal evolution with a final observation is considered. The unique existence of the solution and the first-order optimality condition of the optimal control problem are derived. The convergence of a temporally semidiscrete approximation is rigorously established, where the control is not explicitly discretized and the state equation is discretized by a discontinuous Galerkin method in time. Numerical results are provided to verify the theoretical results.
\end{abstract}

Keywords: Dirichlet boundary control; fractional evolution equation; discontinuous Galerkin method; convergence.

\section{Introduction}

There is an extensive literature on the numerical optimization with PDE constraints. So far, most of the literature focuses on the distributed control problems, and the works on the Dirichlet boundary control problems are rather limited. Compared with the distributed control problems, the Dirichlet boundary control problems are more challenging in the following senses. Firstly, the solution of the state equation of a Dirichlet boundary control problem is of significantly lower regularity than that of a distributed control problem, and this increases the difficulty in both theoretical and numerical analysis. Secondly, in the weak form of the solution of the state equation of a Dirichlet boundary control problem, the test function space is more regular than the trial function space, and hence the weak form is not appropriate for the discretization. Thirdly, since the normal derivative of the adjoint state occurs in the first-order optimality condition, the discrete first-order optimality condition will essentially involve the discrete normal derivative of the discrete adjoint state, and this increases the implementation difficulty.

\footnotetext{
${ }^{*}$ This work was supported by National Natural Science Foundation of China (11901410).

${ }^{\dagger}$ Email:zqmath@aliyun.com

$\ddagger$ Corresponding author. Email: libinjie@scu.edu.cn
} 
We summarize the works on the parabolic Dirichlet boundary control problems briefly as follows. Using an integral representation formula derived by the semigroup theory (cf. [3, Section 4.12] and [30]), Lasiecka [29, 31] analyzed spatial Galerkin approximations of an optimal Dirichlet boundary control problem and a time optimal Dirichlet boundary control problem for the parabolic equations. Kunisch and Vexler [28] analyzed constrained Dirichlet boundary control problems for a class of parabolic equations and derived the convergence of the PDAS strategy for two Dirichlet boundary control problems. Applying the Robin penalization method to a Dirichlet boundary control problem for a parabolic equation with a final observation, Belgacem et al. [4] obtained a penalized Robin boundary control problem. Gong et al. [16] analyzed a finite element approximation of a Dirichlet boundary control for a parabolic equation, where the variational discretization approach [19] was used and the state equation was discretized by the usual $H^{1}$-conforming $P 1$-element in space and discretized by the $\mathrm{dG}(0)$ scheme in time. Recently, Gong and Li [17] improved the spatial accuracy derived in [16], using the maximal $L^{p}$-regularity theory. We note that, for the state equation with rough Dirichlet boundary data, $[29,31]$ used the semigroup theory to define the solution whereas $[16,17,28]$ used the transposition technique to define the solution (called the very weak solution).

For the numerical analysis of parabolic Neumann/Robin boundary control problems, we refer the reader to $[1,7,27,44]$. For the numerical analysis of other optimal control problems for parabolic equations, we refer the reader to $[8,15,34,35,47,48,49,50]$ and the references therein. Although the spatial discretization is not considered in this paper, we would like to refer the reader to $[5,13,14,32]$ for the numerical analysis of elliptic and parabolic equations with rough Dirichlet boundary data.

To our best knowledge, no convergence result is available for the Galerkintype approximations of the Dirichlet boundary control problems governed by the parabolic equations with final observations. The fractional evolution equation is an extension of the normal evolution equation, widely used to describe the physical phenomena with memory effect [55]. Recently, Harbir et al. [2] studied an optimal distributed control problem for a space-time fractional diffusion equation. For the numerical analysis of the optimal distributed problems governed by the time fractional diffusion equations, we refer the reader to [18, 26, 38, 61]. To our knowledge, no numerical analysis is available for the Dirichlet boundary control problems governed by the fractional evolution equations. Hence, this paper tries to analyze the Dirichlet boundary control problems for the fractional and normal evolution equations in a unified way.

In this paper, we establish the convergence of a temporally semidiscrete approximation of an abstract optimal control problem governed by a fractional/normal evolution equation with a final observation. This approximation uses the variational discretization concept [19] and uses a discontinuous Galerkin method to discretize the state equation in time. The discontinuous Galerkin method is the famous $\mathrm{dG}(0)$ scheme for the normal evolution equation, and is equivalent to the well-known L1 scheme [39] with uniform temporal grids for the fractional evolution equations. The derived numerical analysis is applied to a Dirichlet boundary control problem. We note that there are many works (see $[20,21,22,23,25,36,41,43]$ and the references therein) devoted to the numerical analysis of the fractional diffusion equations with rough initial value and source term, but, to our knowledge, no numerical analysis is available for the 
fractional diffusion equation with rough Dirichlet boundary value. This paper also fills in this gap.

The rest of this paper is organized as follows. Section 2 establishes the convergence of a temporally semidiscrete approximation of an abstract optimal control problem. Section 3 applies the theory developed in the previous section to a Dirichlet boundary control problem. Section 4 performs three numerical experiments to confirm the theoretical results.

\section{An abstract optimal control problem}

\subsection{Preliminaries}

We will use the following conventions: for each linear vector space, the field of the scalars is $\mathbb{C}$; for a Hilbert space $\mathcal{X}$, we use $(\cdot, \cdot)_{\mathcal{X}}$ to denote its inner product; for a Banach space $\mathcal{B}$, we use $\langle\cdot, \cdot\rangle_{\mathcal{B}}$ to denote a duality paring between $\mathcal{B}^{*}$ (the dual space of $\mathcal{B}$ ) and $\mathcal{B}$; for a linear operator $A, \rho(A)$ denotes the resolvent set of $A$ and $R(z, A)$ denotes the inverse of $z-A$ for each $z \in \rho(A)$; for two Banach spaces $\mathcal{B}_{1}$ and $\mathcal{B}_{2}, \mathcal{L}\left(\mathcal{B}_{1}, \mathcal{B}_{2}\right)$ is the set of all bounded linear operators from $\mathcal{B}_{1}$ to $\mathcal{B}_{2}$, and $\mathcal{L}\left(\mathcal{B}_{1}, \mathcal{B}_{1}\right)$ is abbreviated to $\mathcal{L}\left(\mathcal{B}_{1}\right) ; I$ denotes the identity map; for a Lebesgue measurable subset $\mathcal{D} \subset \mathbb{R}^{l}, 1 \leqslant l \leqslant 4,\langle p, q\rangle_{\mathcal{D}}$ means the integral $\int_{\mathcal{D}} p \bar{q}$, where $\bar{q}$ is the conjugate of $q$; for a function $v$ defined on $(0, T)$, by $v(t-)$,

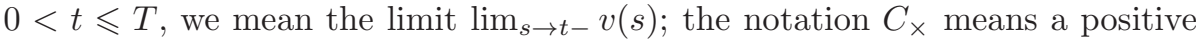
constant, depending only on its subscript(s), and its value may differ at each occurrence; for any $0<\theta<\pi$, define

$$
\begin{aligned}
& \Sigma_{\theta}:=\left\{r e^{i \gamma}: r>0,-\theta<\gamma<\theta\right\}, \\
& \Gamma_{\theta}:=\left\{r e^{-i \theta}: r \geqslant 0\right\} \cup\left\{r e^{i \theta}: r>0\right\} \\
& \Upsilon_{\theta}:=\left\{z \in \Gamma_{\theta}:-\pi \leqslant \Im z \leqslant \pi\right\},
\end{aligned}
$$

where $i$ is the imaginary unit and $\Gamma_{\theta}$ and $\Upsilon_{\theta}$ are so oriented that the negative real axis is to their left.

\subsubsection{Time fractional Sobolev spaces}

Assume that $-\infty<a<b<\infty$ and $\mathcal{B}$ is a Banach space. Define

$$
\begin{aligned}
& { }_{0} H^{1}(a, b ; \mathcal{B}):=\left\{v \in L^{2}(a, b ; \mathcal{B}): v^{\prime} \in L^{2}(a, b ; \mathcal{B}), v(a)=0\right\}, \\
& { }^{0} H^{1}(a, b ; \mathcal{B}):=\left\{v \in L^{2}(a, b ; \mathcal{B}): v^{\prime} \in L^{2}(a, b ; \mathcal{B}), v(b)=0\right\},
\end{aligned}
$$

and endow them with the two norms

$$
\begin{aligned}
& \|v\|_{0 H^{1}(a, b ; \mathcal{B})}:=\left\|v^{\prime}\right\|_{L^{2}(a, b ; \mathcal{B})} \quad \forall v \in{ }_{0} H^{1}(a, b ; \mathcal{B}), \\
& \|v\|_{{ }_{0} H^{1}(a, b ; \mathcal{B})}:=\left\|v^{\prime}\right\|_{L^{2}(a, b ; \mathcal{B})} \quad \forall v \in{ }^{0} H^{1}(a, b ; \mathcal{B}),
\end{aligned}
$$

respectively, where $v^{\prime}$ is the first-order weak derivative of $v$.

For each $0<\gamma<1$, define

$$
\begin{aligned}
& { }_{0} H^{\gamma}(a, b ; \mathcal{B}):=\left(L^{2}(a, b ; \mathcal{B}),{ }_{0} H^{1}(a, b ; \mathcal{B})\right)_{\gamma, 2}, \\
& { }^{0} H^{\gamma}(a, b ; \mathcal{B}):=\left(L^{2}(a, b ; \mathcal{B}),{ }^{0} H^{1}(a, b ; \mathcal{B})\right)_{\gamma, 2},
\end{aligned}
$$

where $(\cdot, \cdot)_{\gamma, 2}$ means the interpolation space defined by the $K$-method (cf. [42]). For convenience, the spaces ${ }_{0} H^{\gamma}(a, b ; \mathbb{C})$ and ${ }^{0} H^{\gamma}(a, b ; \mathbb{C})$ will be abbreviated to ${ }_{0} H^{\gamma}(a, b)$ and ${ }^{0} H^{\gamma}(a, b)$, respectively. 


\subsubsection{Riemann-Liouville fractional calculus operators}

Assume that $-\infty<a<b<\infty$ and $\mathcal{X}$ is a separable Hilbert space. For any $0<\gamma<1$, define

$$
\begin{array}{ll}
\left(\mathrm{D}_{a+}^{-\gamma} v\right)(t):=\frac{1}{\Gamma(\gamma)} \int_{a}^{t}(t-s)^{\gamma-1} v(s) \mathrm{d} s, & \text { a.e. } t \in(a, b), \\
\left(\mathrm{D}_{b-}^{-\gamma} v\right)(t):=\frac{1}{\Gamma(\gamma)} \int_{t}^{b}(s-t)^{\gamma-1} v(s) \mathrm{d} s, & \text { a.e. } t \in(a, b),
\end{array}
$$

for all $v \in L^{1}(a, b ; \mathcal{X})$, where $\Gamma(\cdot)$ is the gamma function. In addition, let $\mathrm{D}_{a+}^{0}$ and $\mathrm{D}_{b-}^{0}$ be the identity operator on $L^{1}(a, b ; \mathcal{X})$. For any $0<\gamma \leqslant 1$, define

$$
\begin{aligned}
& \mathrm{D}_{a+}^{\gamma} v:=\mathrm{D} \mathrm{D}_{a+}^{\gamma-1} v, \\
& \mathrm{D}_{b-}^{\gamma} v:=-\mathrm{D} \mathrm{D}_{b-}^{\gamma-1} v,
\end{aligned}
$$

for all $v \in L^{1}(a, b ; \mathcal{X})$, where $\mathrm{D}$ is the first-order differential operator in the distribution sense. have

Assume that $0<\gamma<1$. For any $v \in{ }_{0} H^{\gamma}(a, b ; \mathcal{X})$ and $w \in{ }^{0} H^{\gamma}(a, b ; \mathcal{X})$, we

$$
\begin{aligned}
& C_{1}\|v\|_{0^{\gamma}(a, b ; \mathcal{X})} \leqslant\left\|\mathrm{D}_{a+}^{\gamma} v\right\|_{L^{2}(a, b ; \mathcal{X})} \leqslant C_{2}\|v\|_{0^{\gamma} H^{\gamma}(a, b ; \mathcal{X})}, \\
& C_{1}\|w\|_{0_{H^{\gamma}(a, b ; \mathcal{X})}} \leqslant\left\|\mathrm{D}_{b-}^{\gamma} w\right\|_{L^{2}(a, b ; \mathcal{X})} \leqslant C_{2}\|w\|_{{ }_{0} H^{\gamma}(a, b ; \mathcal{X})},
\end{aligned}
$$

where $C_{1}$ and $C_{2}$ are two positive constants depending only on $\gamma$. Let $\mathcal{X}^{*}$ be the dual space of $\mathcal{X}$. For any $v \in{ }_{0} H^{\gamma / 2}\left(a, b ; \mathcal{X}^{*}\right)$ and $w \in{ }^{0} H^{\gamma / 2}(a, b ; \mathcal{X})$, the equality

$$
\int_{0}^{T}\left\langle\mathrm{D}_{a+}^{\gamma} v, w\right\rangle_{\mathcal{X}} \mathrm{d} t=\int_{0}^{T}\left\langle v, \mathrm{D}_{b-}^{\gamma} w\right\rangle_{\mathcal{X}} \mathrm{d} t
$$

holds for the following two cases: $v \in{ }_{0} H^{\gamma}\left(a, b ; \mathcal{X}^{*}\right)$ and $w \in{ }^{0} H^{\gamma}(a, b ; \mathcal{X})$; $\mathrm{D}_{a+}^{\gamma} v \in L^{2 /(1+\gamma)}\left(a, b ; \mathcal{X}^{*}\right)$ and $\mathrm{D}_{b-}^{\gamma} v \in L^{2 /(1+\gamma)}(a, b ; \mathcal{X})$. For the above theoretical results, we refer the reader to $[11,43]$.

\subsubsection{Definitions of $\mathcal{A}$ and $\mathcal{A}^{*}$}

Assume that $X$ and $Y$ are two separable Hilbert spaces such that $X$ is continuously embedded into $Y$ and $X$ is dense in $Y$. We will regard $Y$ as a subspace of $X^{*}$, the dual space of $X$, in the sense that

$$
\langle v, w\rangle_{X}:=(v, w)_{Y} \quad \text { for all } v \in Y \text { and } w \in X .
$$

Let $\mathcal{A}$ and $\mathcal{A}^{*}$ be two bounded linear operators from $X$ to $Y$ satisfying that

$$
\left\{\begin{array}{l}
\rho(\mathcal{A}) \supset \Sigma_{\omega_{0}} \cup\{0\}, \quad \rho\left(\mathcal{A}^{*}\right) \supset \Sigma_{\omega_{0}} \cup\{0\}, \\
\|R(z, \mathcal{A})\|_{\mathcal{L}(Y)} \leqslant \frac{\mathcal{M}_{0}}{1+|z|} \quad \forall z \in \Sigma_{\omega_{0}}, \\
\left\|R\left(z, \mathcal{A}^{*}\right)\right\|_{\mathcal{L}(Y)} \leqslant \frac{\mathcal{M}_{0}}{1+|z|} \quad \forall z \in \Sigma_{\omega_{0}}, \\
(\mathcal{A} v, w)_{Y}=\left(v, \mathcal{A}^{*} w\right)_{Y} \quad \forall v, w \in X, \\
c_{0}\|v\|_{X} \leqslant\|\mathcal{A} v\|_{Y} \leqslant c_{1}\|v\|_{X} \quad \forall v \in X, \\
c_{0}\|v\|_{X} \leqslant\left\|\mathcal{A}^{*} v\right\|_{Y} \leqslant c_{1}\|v\|_{X} \quad \forall v \in X, \\
(\mathcal{A} v, v)_{Y} \geqslant 0 \quad \forall v \in X,
\end{array}\right.
$$


where $\pi / 2<\omega_{0}<\pi, c_{0}, c_{1}$ and $\mathcal{M}_{0}$ are four positive constants. By the transposition technique, $\mathcal{A}$ and $\mathcal{A}^{*}$ can be extended as two bounded linear operators from $Y$ to $X^{*}$ by

$$
\begin{aligned}
\langle\mathcal{A} v, w\rangle_{X} & :=\left(v, \mathcal{A}^{*} w\right)_{Y}, \\
\left\langle\mathcal{A}^{*} v, w\right\rangle_{X} & :=(v, \mathcal{A} w)_{Y},
\end{aligned}
$$

for all $v \in Y$ and $w \in X$.

For each $0 \leqslant \theta \leqslant 1$, let $\left[X^{*}, Y\right]_{\theta}$ and $[X, Y]_{\theta}$ be the interpolation spaces defined by the famous complex interpolation method (cf. [42, Chapter 2]). We have that $\left[X^{*}, Y\right]_{\theta}$ is the dual space of $[X, Y]_{\theta}$ and vice versa (cf. [6]). By (5e), (5f) and [42, Theorem 2.6], a straightforward computation gives that

$$
\|\mathcal{A}\|_{\mathcal{L}\left([X, Y]_{\theta},\left[X^{*}, Y\right]_{1-\theta}\right)} \leqslant c_{1} \text { for all } 0 \leqslant \theta \leqslant 1 .
$$

Lemma 2.1. Assume that $0 \leqslant \theta \leqslant 1$ and $z \in \Sigma_{\omega_{0}}$. Then

$$
\begin{aligned}
\|R(z, \mathcal{A})\|_{\mathcal{L}\left(Y,[X, Y]_{\theta}\right)} & \leqslant \frac{C_{c_{0}, \mathcal{M}_{0}}}{1+|z|^{\theta}}, \\
\left\|R\left(z, \mathcal{A}^{*}\right)\right\|_{\mathcal{L}\left(Y,[X, Y]_{\theta}\right)} & \leqslant \frac{C_{c_{0}, \mathcal{M}_{0}}}{1+|z|^{\theta}}, \\
\|R(z, \mathcal{A})\|_{\mathcal{L}\left(\left[X^{*}, Y\right]_{\theta}, Y\right)} & \leqslant \frac{C_{c_{0}, \mathcal{M}_{0}}}{1+|z|^{\theta}}, \\
\left\|R\left(z, \mathcal{A}^{*}\right)\right\|_{\mathcal{L}\left(\left[X^{*}, Y\right]_{\theta}, Y\right)} & \leqslant \frac{C_{c_{0}, \mathcal{M}_{0}}}{1+|z|^{\theta}} .
\end{aligned}
$$

Moreover, for any $0 \leqslant \epsilon \leqslant 1$,

$$
\|R(z, \mathcal{A})\|_{\mathcal{L}\left(\left[X^{*}, Y\right]_{\theta},[X, Y]_{1-(1-\epsilon) \theta}\right)} \leqslant \frac{C_{c_{0}, \mathcal{M}_{0}, \epsilon, \theta}}{1+|z|^{\epsilon \theta}} .
$$

Proof. A straightforward computation gives

$$
\|\mathcal{A} R(z, \mathcal{A})\|_{\mathcal{L}(Y)}=\|z R(z, \mathcal{A})-I\|_{\mathcal{L}(Y)} \leqslant|z|\|R(z, \mathcal{A})\|_{\mathcal{L}(Y)}+1 \leqslant 1+\mathcal{M}_{0},
$$

by $(5 \mathrm{~b})$, so that (5e) implies

$$
\|R(z, \mathcal{A})\|_{\mathcal{L}(Y, X)} \leqslant \frac{1+\mathcal{M}_{0}}{c_{0}} .
$$

By this estimate and (5b), using [42, Theorem 2.6] yields (9). Estimate (10) can be proved analogously.

Then let us prove (11). We first consider the following problem: seek $v \in Y$ such that

$$
\left(v,\left(\bar{z}-\mathcal{A}^{*}\right)_{\varphi}\right)_{Y}=\langle g, \varphi\rangle_{X} \quad \forall \varphi \in X,
$$

where $g \in X^{*}$ is arbitrary but fixed. By (5f) and the fact that $X$ is continuously embedded into $Y$, we conclude that $\left(\cdot,\left(\bar{z}-\mathcal{A}^{*}\right) \cdot\right)_{Y}$ is a continuous bilinear form on $Y \times X$. Inserting $\theta=0$ into (10) implies that, for any $v \in Y \backslash\{0\}$,

$$
\sup _{\varphi \in X \backslash\{0\}} \frac{\left|\left(v,\left(\bar{z}-\mathcal{A}^{*}\right) \varphi\right)_{Y}\right|}{\|\varphi\|_{X}} \geqslant \frac{\|v\|_{Y}^{2}}{\left\|R\left(\bar{z}, \mathcal{A}^{*}\right) v\right\|_{X}} \geqslant C_{c_{0}, \mathcal{M}_{0}}\|v\|_{Y} .
$$


Since $z \in \rho\left(\mathcal{A}^{*}\right)$, it is evident that, for any $\varphi \in X \backslash\{0\}$,

$$
\sup _{v \in Y}\left|\left(v,\left(\bar{z}-\mathcal{A}^{*}\right) \varphi\right)_{Y}\right|>0 .
$$

Consequently, the Babuska-Lax-Milgram theorem yields that problem (14) admits a unique solution $v \in Y$ and $\|v\|_{Y} \leqslant C_{c_{0}, \mathcal{M}_{0}}\|g\|_{X^{*}}$. Since (6) and (14) imply $v=R(z, \mathcal{A}) g$, this indicates that

$$
\|R(z, A) g\|_{Y} \leqslant C_{c_{0}, \mathcal{M}_{0}}\|g\|_{X^{*}} \quad \forall g \in X^{*}
$$

and hence

$$
\|R(z, A)\|_{\mathcal{L}\left(X^{*}, Y\right)} \leqslant C_{c_{0}, \mathcal{M}_{0}} .
$$

By (5b) and (15), using [42, Theorem 2.6] yields (11). Estimate (12) is derived similarly.

Finally, let us prove (13). Inserting $\theta=\epsilon$ and $\theta=0$ into (9) and (11) respectively yields

$$
\|R(z, \mathcal{A})\|_{\mathcal{L}\left(Y,[X, Y]_{\epsilon}\right)} \leqslant \frac{C_{c_{0}, \mathcal{M}_{0}}}{1+|z|^{\epsilon}}, \quad\|R(z, \mathcal{A})\|_{\mathcal{L}\left(X^{*}, Y\right)} \leqslant C_{c_{0}, \mathcal{M}_{0}} .
$$

Using [42, Theorem 2.6] then gives

$$
\|R(z, \mathcal{A})\|_{\mathcal{L}\left(\left[X^{*}, Y\right]_{\theta},\left[Y,[X, Y]_{\epsilon}\right]_{\theta}\right)} \leqslant \frac{C_{c_{0}, \mathcal{M}_{0}}}{1+\left.|z|\right|^{\epsilon \theta}} .
$$

Hence, by the fact that (cf. [6])

$$
\left[Y,[X, Y]_{\epsilon}\right]_{\theta}=[X, Y]_{1-(1-\epsilon) \theta} \text { with equivalent norms, }
$$

we readily obtain (13). This completes the proof.

Remark 2.1. For any $z \in \Sigma_{\omega_{0}}$,

$$
\begin{aligned}
\|R(z, \mathcal{A})\|_{\mathcal{L}\left(X^{*}\right)} & =\left\|z^{-1}(z-\mathcal{A}+\mathcal{A}) R(z, \mathcal{A})\right\|_{\mathcal{L}\left(X^{*}\right)} \\
& =\|I+\mathcal{A} R(z, \mathcal{A})\|_{\mathcal{L}\left(X^{*}\right)} /|z| \\
& \leqslant \frac{1+\|\mathcal{A} R(z, \mathcal{A})\|_{\mathcal{L}\left(X^{*}\right)}}{|z|} \\
& \leqslant \frac{1+c_{1}\|R(z, \mathcal{A})\|_{\mathcal{L}\left(X^{*}, Y\right)}}{|z|} \quad \text { (by inserting } \theta=1 \text { into } \text { (8)) } \\
& \leqslant \frac{C_{c_{0}, c_{1}, \mathcal{M}_{0}}}{|z|} \quad \text { (by inserting } \theta=0 \text { into (11)). }
\end{aligned}
$$

Also, we have

$$
\begin{aligned}
\|R(z, \mathcal{A})\|_{\mathcal{L}\left(X^{*}\right)} & =\left\|\mathcal{A} \mathcal{A}^{-1} R(z, \mathcal{A})\right\|_{\mathcal{L}\left(X^{*}\right)} \\
& \left.\leqslant c_{1}\left\|\mathcal{A}^{-1} R(z, \mathcal{A})\right\|_{\mathcal{L}\left(X^{*}, Y\right)} \quad \text { (by inserting } \theta=1 \text { into }(8)\right) \\
& \leqslant C_{c_{1}, \mathcal{M}_{0}}\|R(z, \mathcal{A})\|_{\mathcal{L}\left(X^{*}, Y\right)} \quad(\text { by }(5 \mathrm{~b})) \\
& \left.\leqslant C_{c_{0}, c_{1}, \mathcal{M}_{0}} \quad \text { (by inserting } \theta=0 \text { into }(11)\right) .
\end{aligned}
$$

Consequently,

$$
\|R(z, \mathcal{A})\|_{\mathcal{L}\left(X^{*}\right)} \leqslant \frac{C_{c_{0}, c_{1}, \mathcal{M}_{0}}}{1+|z|} \quad \forall z \in \Sigma_{\omega_{0}} .
$$




\subsubsection{Definitions of $E_{\alpha}$ and $E_{\alpha}^{*}$}

For any $0<\alpha \leqslant 1$ and $t>0$, define

$$
\begin{aligned}
& E_{\alpha}(t):=\frac{1}{2 \pi i} \int_{\Gamma_{\omega_{0}}} e^{t z} R\left(z^{\alpha}, \mathcal{A}\right) \mathrm{d} z \\
& E_{\alpha}^{*}(t):=\frac{1}{2 \pi i} \int_{\Gamma_{\omega_{0}}} e^{t \bar{z}} R\left(\overline{z^{\alpha}}, \mathcal{A}^{*}\right) \overline{\mathrm{d} z}
\end{aligned}
$$

For any $v \in X^{*}$ and $w \in Y$, by the definitions of $\mathcal{A}$ and $\mathcal{A}^{*}$ and Lemma 2.1 we have that

$$
(R(z, \mathcal{A}) v, w)_{Y}=\left\langle v, R\left(\bar{z}, \mathcal{A}^{*}\right) w\right\rangle_{X} \quad \forall z \in \Sigma_{\omega_{0}},
$$

and hence from (17) and (18) we obtain

$$
\left(E_{\alpha}(t) v, w\right)_{Y}=\left\langle v, E_{\alpha}^{*}(t) w\right\rangle_{X} \quad \forall t>0 .
$$

Furthermore, by Lemma 2.1, a routine calculation (cf. [59, 23]) yields the following lemma.

Lemma 2.2. Assume that $0<\alpha \leqslant 1,0 \leqslant \theta \leqslant 1, t>0$, and $G=E_{\alpha}$ or $E_{\alpha}^{*}$. Then

$$
\begin{aligned}
\|G(t)\|_{\mathcal{L}\left(Y,[X, Y]_{\theta}\right)} & \leqslant C_{c_{0}, \omega_{0}, \mathcal{M}_{0}} t^{\theta \alpha-1} \\
\|G(t)\|_{\mathcal{L}\left(\left[X^{*}, Y\right]_{\theta}, Y\right)} & \leqslant C_{c_{0}, \omega_{0}, \mathcal{M}_{0}} t^{\theta \alpha-1} \\
\left\|G^{\prime}(t)\right\|_{\mathcal{L}\left(Y,[X, Y]_{\theta}\right)} & \leqslant C_{c_{0}, \omega_{0}, \mathcal{M}_{0}} t^{\theta \alpha-2} \\
\left\|G^{\prime}(t)\right\|_{\mathcal{L}\left(\left[X^{*}, Y\right]_{\theta}, Y\right)} & \leqslant C_{c_{0}, \omega_{0}, \mathcal{M}_{0}} t^{\theta \alpha-2}
\end{aligned}
$$

Moreover, for any $0 \leqslant \epsilon \leqslant 1$,

$$
E_{\alpha} \in C\left((0, \infty) ; \mathcal{L}\left(\left[X^{*}, Y\right]_{\theta},[X, Y]_{1-(1-\epsilon) \theta}\right)\right.
$$

and

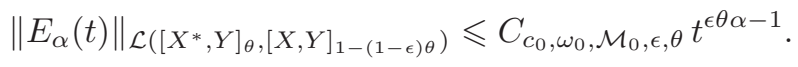

Remark 2.2. By (16) we have

$$
\left\|E_{\alpha}(t)\right\|_{\mathcal{L}\left(X^{*}\right)} \leqslant C_{c_{0}, c_{1}, \omega_{0}, \mathcal{M}_{0}} t^{\alpha-1}, \quad \forall t>0 .
$$

\subsubsection{Solutions of the fractional evolution equations}

Following the mild solution theory of fractional/normal evolution equations in $[54,41,46,23]$, we introduce the following mild solutions. Assume that $0<\alpha \leqslant$ 1 and $0<T<\infty$. For any

$$
g \in L^{1}\left(0, T ;\left[X^{*}, Y\right]_{\theta}\right) \quad \text { with } \quad 0 \leqslant \theta \leqslant 1,
$$

we call

$$
\left(\mathcal{S}_{\alpha} g\right)(t):=\int_{0}^{t} E_{\alpha}(t-s) g(s) \mathrm{d} s, \quad \text { a.e. } 0<t \leqslant T,
$$

the mild solution to the following fractional evolution equation:

$$
\left(\mathrm{D}_{0+}^{\alpha}-\mathcal{A}\right) w=g, \quad w(0)=0 .
$$


For any $v \in\left[X^{*}, Y\right]_{\theta}, 0 \leqslant \theta \leqslant 1$, we call

$$
\left(\mathcal{S}_{\alpha}\left(v \delta_{0}\right)\right)(t):=E_{\alpha}(t) v, \quad 0<t \leqslant T,
$$

the mild solution to (27) with $g=v \delta_{0}$, where $\delta_{0}$ is the Dirac measure in time concentrated at $t=0$. Symmetrically, for any

$$
g \in L^{1}\left(0, T ;\left[X^{*}, Y\right]_{\theta}\right) \text { with } \quad 0 \leqslant \theta \leqslant 1
$$

we call

$$
\left(\mathcal{S}_{\alpha}^{*} g\right)(t):=\int_{t}^{T} E_{\alpha}^{*}(s-t) g(s) \mathrm{d} s, \quad \text { a.e. } 0<t<T,
$$

the mild solution to the following backward fractional evolution equation:

$$
\left(\mathrm{D}_{T-}^{\alpha}-\mathcal{A}^{*}\right) w=g, \quad w(T)=0 .
$$

For any $v \in\left[X^{*}, Y\right]_{\theta}, 0 \leqslant \theta \leqslant 1$, we call

$$
\left(\mathcal{S}_{\alpha}^{*}\left(v \delta_{T}\right)\right)(t):=E_{\alpha}^{*}(T-t) v, \quad 0<t \leqslant T,
$$

the mild solution to equation (30) with $g=v \delta_{T}$, where $\delta_{T}$ is the Dirac measure in time concentrated at $t=T$.

Lemma 2.3. Assume that $0<\alpha, \theta \leqslant 1$ and $q>1 /(\theta \alpha)$. Then

$$
\begin{aligned}
& \mathcal{S}_{\alpha} \in \mathcal{L}\left(L^{2}\left(0, T ;\left[X^{*}, Y\right]_{\theta}\right), L^{2}(0, T ; Y)\right), \\
& \mathcal{S}_{\alpha} \in \mathcal{L}\left(L^{q}\left(0, T ;\left[X^{*}, Y\right]_{\theta}\right), C([0, T] ; Y)\right) .
\end{aligned}
$$

Moreover, for any $g \in L^{q}\left(0, T ;\left[X^{*}, Y\right]_{\theta}\right)$ and $v \in Y$,

$$
\left(\left(\mathcal{S}_{\alpha} g\right)(T), v\right)_{Y}=\int_{0}^{T}\left\langle g(t),\left(\mathcal{S}_{\alpha}^{*}\left(v \delta_{T}\right)\right)(t)\right\rangle_{X} \mathrm{~d} t .
$$

Proof. By (21) and (26), a routine argument (cf. [9, Theorem 2.6]) yields (32) and (33). Note that (26) and (33) imply

$$
\left(\mathcal{S}_{\alpha} g\right)(T)=\int_{0}^{T} E_{\alpha}(T-t) g(t) \mathrm{d} t
$$

and hence

$$
\begin{aligned}
\left(\left(\mathcal{S}_{\alpha} g\right)(T), v\right)_{Y} & =\left(\int_{0}^{T} E_{\alpha}(T-t) g(t) \mathrm{d} t, v\right)_{Y}=\int_{0}^{T}\left(E_{\alpha}(T-t) g(t), v\right)_{Y} \mathrm{~d} t \\
& =\int_{0}^{T}\left\langle g(t), E_{\alpha}^{*}(T-t) v\right\rangle_{X} \mathrm{~d} t \quad(\text { by }(19)) \\
& =\int_{0}^{T}\left\langle g(t),\left(\mathcal{S}_{\alpha}^{*}\left(v \delta_{T}\right)\right)(t)\right\rangle_{X} \mathrm{~d} t \quad(\text { by }(31)) .
\end{aligned}
$$

This proves (34) and hence this lemma.

Lemma 2.4. For any $0<\theta \leqslant 1$,

$$
\begin{gathered}
\lim _{\alpha \rightarrow 1-}\left\|\mathcal{S}_{\alpha}-\mathcal{S}_{1}\right\|_{\mathcal{L}\left(L^{1}\left(0, T ;\left[X^{*}, Y\right]_{\theta}\right), L^{1}(0, T ; Y)\right)}=0 \\
\lim _{\alpha \rightarrow 1-}\left\|\mathcal{S}_{\alpha}-\mathcal{S}_{1}\right\|_{\mathcal{L}\left(L^{\infty}\left(0, T ;\left[X^{*}, Y\right]_{\theta}\right), C([0, T] ; Y)\right)}=0 .
\end{gathered}
$$


Proof. Since

$$
R(z, \mathcal{A})-R\left(z^{\alpha}, \mathcal{A}\right)=\left(z^{\alpha}-z\right) R(z, \mathcal{A}) R\left(z^{\alpha}, \mathcal{A}\right) \quad \text { for all } z \in \Sigma_{\omega_{0}},
$$

a straightforward calculation gives, by (17) and Lemma 2.1, that

$$
\left\|\left(E_{\alpha}-E_{1}\right)(t)\right\|_{\mathcal{L}\left(\left[X^{*}, Y\right]_{\theta}, Y\right)} \leqslant C_{c_{0}, \mathcal{M}_{0}} \int_{0}^{\infty} e^{t \cos \omega_{0} r} \frac{\left|r e^{i \omega_{0}}-\left(r e^{i \omega_{0}}\right)^{\alpha}\right|}{(1+r)\left(1+r^{\theta \alpha}\right)} \mathrm{d} r
$$

for all $t>0$. It follows that

$$
\left\|E_{\alpha}-E_{1}\right\|_{L^{1}\left(0, T ; \mathcal{L}\left(\left[X^{*}, Y\right]_{\theta}, Y\right)\right)} \leqslant C_{c_{0}, \omega_{0}, \mathcal{M}_{0}} \int_{0}^{\infty} \frac{\left|r e^{i \omega_{0}}-\left(r e^{i \omega_{0}}\right)^{\alpha}\right|}{r(1+r)\left(1+r^{\theta \alpha}\right)} \mathrm{d} r .
$$

Then Lebesgue's dominated convergence theorem yields

$$
\lim _{\alpha \rightarrow 1-}\left\|E_{\alpha}-E_{1}\right\|_{L^{1}\left(0, T ; \mathcal{L}\left(\left[X^{*}, Y\right]_{\theta}, Y\right)\right)}=0 .
$$

Since Young's inequality implies

$$
\left\|\mathcal{S}_{\alpha}-\mathcal{S}_{1}\right\|_{\mathcal{L}\left(L^{1}\left(0, T ;\left[X^{*}, Y\right]_{\theta}\right), L^{1}(0, T ; Y)\right.} \leqslant\left\|\mathcal{E}_{\alpha}-E_{1}\right\|_{L^{1}\left(0, T ; \mathcal{L}\left(\left[X^{*}, Y\right]_{\theta}\right)\right)},
$$

by (37) we readily obtain (35). Moreover, by (26) and (33) we have

$$
\left\|\mathcal{S}_{\alpha}-\mathcal{S}_{1}\right\|_{\mathcal{L}\left(L^{\infty}\left(0, T ;\left[X^{*}, Y\right]_{\theta}, Y\right), C([0, T] ; Y)\right)} \leqslant\left\|E_{\alpha}-E_{1}\right\|_{L^{1}\left(0, T ; \mathcal{L}\left(\left[X^{*}, Y\right]_{\theta}, Y\right)\right)},
$$

so that (37) proves (36). This completes the proof.

Lemma 2.5. Assume that $0<\alpha, \theta \leqslant 1$. Then for any $g \in C\left([0, T] ;\left[X^{*}, Y\right]_{\theta}\right)$ we have

$$
\left(\mathrm{D}_{0+}^{\alpha}-\mathcal{A}\right) \mathcal{S}_{\alpha} g=g
$$

and

$$
\begin{aligned}
& \left\|\mathrm{D}_{0+}^{\alpha} \mathcal{S}_{\alpha} g\right\|_{C\left([0, T] ;\left[X^{*}, Y\right]_{(1-\epsilon) \theta}\right)}+\left\|\mathcal{A} \mathcal{S}_{\alpha} g\right\|_{C\left([0, T] ;\left[X^{*}, Y\right]_{(1-\epsilon) \theta}\right)} \\
\leqslant & c\|g\|_{C\left([0, T] ;\left[X^{*}, Y\right]_{\theta}\right)},
\end{aligned}
$$

where $0<\epsilon<1$ and $c$ is a positive constant independent of $g$.

Proof. Since a complete rigorous proof of this lemma is tedious but standard (cf. [54]), we only present briefly the key ingredients of the proof.

Step 1. Define

$$
\eta(t):=\frac{1}{2 \pi i} \int_{\Gamma_{\omega_{0}}} e^{t z} z^{\alpha-1} R\left(z^{\alpha}, \mathcal{A}\right) \mathrm{d} z, \quad t>0 .
$$

A straightforward computation gives that, for any $0<t \leqslant T$,

$$
\begin{aligned}
\eta(t) & =\frac{1}{2 \pi i} \int_{\Gamma_{\omega_{0}}} e^{t z} z^{-1}\left(z^{\alpha}-\mathcal{A}+\mathcal{A}\right) R\left(z^{\alpha}, \mathcal{A}\right) \mathrm{d} z \\
& =\frac{1}{2 \pi i} \int_{\Gamma_{\omega_{0}}} e^{t z} z^{-1} I \mathrm{~d} z+\frac{1}{2 \pi i} \int_{\Gamma_{\omega_{0}}} e^{t z} z^{-1} \mathcal{A} R\left(z^{\alpha}, \mathcal{A}\right) \mathrm{d} z \\
& =I+\frac{1}{2 \pi i} \mathcal{A} \int_{\Gamma_{\omega_{0}}} e^{t z} z^{-1} R\left(z^{\alpha}, \mathcal{A}\right) \mathrm{d} z
\end{aligned}
$$


where $\Gamma_{\omega_{0}}$ is deformed so that the origin is to its left. Hence, we conclude from (8) and Lemma 2.1 the following properties:

$$
\begin{aligned}
& \eta \in C\left([0, T] ; \mathcal{L}\left(\left[X^{*}, Y\right]_{\theta}, X^{*}\right)\right) \cap C^{1}\left((0, T] ; \mathcal{L}\left(\left[X^{*}, Y\right]_{\theta}, X^{*}\right)\right) ; \\
& \eta(0)=I \\
& \eta^{\prime}(t)=\frac{1}{2 \pi i} \mathcal{A} \int_{\Gamma_{\omega_{0}}} e^{t z} R\left(z^{\alpha}, \mathcal{A}\right) \mathrm{d} z, \quad t>0 ; \\
& \left\|\eta^{\prime}(t)\right\|_{\mathcal{L}\left(\left[X^{*}, Y\right]_{\theta}, X^{*}\right)} \leqslant C_{c_{0}, c_{1}, \omega_{0}, \mathcal{M}_{0}} \theta^{\theta \alpha-1}, \quad t>0 .
\end{aligned}
$$

Step 2. By the theory of Laplace transform, from (26) we obtain that

$$
\left(\mathrm{D}_{0+}^{\alpha-1} \mathcal{S}_{\alpha} g\right)(t)=\int_{0}^{t} \eta(t-s) g(s) \mathrm{d} s, \quad 0<t \leqslant T .
$$

Hence, by the properties of $\eta$ presented in Step 1,

$$
\begin{aligned}
& \left(\mathrm{D}_{0+}^{\alpha} \mathcal{S}_{\alpha} g\right)(t)=\frac{\mathrm{d}}{\mathrm{d} t}\left(\mathrm{D}_{0+}^{\alpha-1} \mathcal{S}_{\alpha} g\right)(t)=\frac{\mathrm{d}}{\mathrm{d} t} \int_{0}^{t} \eta(t-s) g(s) \mathrm{d} s \\
= & g(t)+\int_{0}^{t} \eta^{\prime}(t-s) g(s) \mathrm{d} s \\
= & g(t)+\mathcal{A} \int_{0}^{t} \frac{1}{2 \pi i} \int_{\Gamma_{\omega_{0}}} e^{(t-s) z} R\left(z^{\alpha}, \mathcal{A}\right) \mathrm{d} z g(s) \mathrm{d} s \\
= & g(t)+\mathcal{A} \int_{0}^{t} E_{\alpha}(t-s) g(s) \mathrm{d} s \quad(\text { by }(17)) \\
= & g(t)+\mathcal{A}\left(\mathcal{S}_{\alpha} g\right)(t) \quad(\text { by }(26))
\end{aligned}
$$

for each $0 \leqslant t \leqslant T$. This implies equality (38).

Step 3. For convenience, we will use $c$ to denote a positive constant, whose value is independent of $g$ but may differ at each occurrence. A routine calculation gives, by (24), (25) and (26), that

$$
\left\|\mathcal{S}_{\alpha} g\right\|_{C\left([0, T] ;[X, Y]_{1-(1-\epsilon) \theta}\right)} \leqslant c\|g\|_{C\left([0, T] ;\left[X^{*}, Y\right]_{\theta}\right)},
$$

so that (8) implies

$$
\left\|\mathcal{A} \mathcal{S}_{\alpha} g\right\|_{C\left([0, T] ;\left[X^{*}, Y\right]_{(1-\epsilon) \theta}\right)} \leqslant c\|g\|_{C\left([0, T] ;\left[X^{*}, Y\right]_{\theta}\right)} .
$$

Since $\left[X^{*}, Y\right]_{\theta}$ is continuously embedded into $\left[X^{*}, Y\right]_{(1-\epsilon) \theta}$, we have

$$
\|g\|_{C\left([0, T] ;\left[X^{*}, Y\right]_{(1-\epsilon) \theta}\right)} \leqslant c\|g\|_{C\left([0, T] ;\left[X^{*}, Y\right]_{\theta}\right)} .
$$

Combining (38), (42) and (43) proves (39) and thus concludes the proof.

\subsection{Continuous problem}

Let $Z$ be a Hilbert space and let $U_{\text {ad }} \subset L^{\infty}(0, T ; Z)$ be a convex, bounded and closed subset of $L^{2}(0, T ; Z)$. We consider the following abstract optimal control problem:

$$
\min _{u \in U_{\text {ad }}} J_{\alpha}(u):=\frac{1}{2}\left\|\left(\mathcal{S}_{\alpha} \mathcal{R}_{\theta_{0}} u\right)(T)-y_{d}\right\|_{Y}^{2}+\frac{\nu}{2}\|u\|_{L^{2}(0, T ; Z)}^{2},
$$


where $0<\alpha \leqslant 1, y_{d} \in Y, \nu>0$ is a regularization parameter, and $\mathcal{R}_{\theta_{0}}: Z \rightarrow$ $\left[X^{*}, Y\right]_{\theta_{0}}$ is a bounded linear operator for some $0<\theta_{0} \leqslant 1$.

Define $\mathcal{R}_{\theta_{0}}^{*}:[X, Y]_{\theta_{0}} \rightarrow Z$ by

$$
\left(\mathcal{R}_{\theta_{0}}^{*} v, w\right)_{Z}:=\overline{\left\langle\mathcal{R}_{\theta_{0}} w, v\right\rangle_{[X, Y]_{\theta_{0}}}}
$$

for all $v \in[X, Y]_{\theta_{0}}$ and $w \in Z$. Assume that $q>\max \left\{1 /\left(\theta_{0} \alpha\right), 2\right\}$. By (33), $\left(\mathcal{S}_{\alpha} \mathcal{R}_{\theta_{0}} \cdot\right)(T)$ is a bounded linear operator from $L^{q}(0, T ; Z)$ to $Y$. Clearly, $J_{\alpha}$ in (44) is a strictly convex functional on $L^{q}(0, T ; Z)$, and $U_{\text {ad }}$ is a convex, bounded and closed subset of $L^{q}(0, T ; Z)$. By (34), a routine argument (cf. [60, Theorems 2.14 and 2.21]) yields the following theorem.

Theorem 2.1. Problem (44) admits a unique solution $u \in U_{\text {ad }}$, and the following first-order optimality condition holds:

$$
\left\{\begin{array}{l}
y=\mathcal{S}_{\alpha} \mathcal{R}_{\theta_{0}} u, \\
p=\mathcal{S}_{\alpha}^{*}\left(\left(y(T)-y_{d}\right) \delta_{T}\right), \\
\int_{0}^{T}\left(\mathcal{R}_{\theta_{0}}^{*} p(t)+\nu u(t), v(t)-u(t)\right)_{Z} \mathrm{~d} t \geqslant 0 \text { for all } v \in U_{a d} .
\end{array}\right.
$$

\subsection{Temporally discrete problem}

Let $J>1$ be an integer and define $t_{j}:=j \tau$ for each $j=0,1,2, \ldots, J$, where $\tau:=T / J$. For each Banach space $\mathcal{X}$, define

$$
W_{\tau}(\mathcal{X}):=\left\{V \in L^{\infty}(0, T ; \mathcal{X}): V \text { is constant on }\left(t_{j-1}, t_{j}\right) \quad \forall 1 \leqslant j \leqslant J\right\} .
$$

For any $0<\alpha<1$ and $g \in L^{1}\left(0, T ; X^{*}\right)$, define $\mathcal{S}_{\alpha, \tau} g \in W_{\tau}(Y)$ and $\mathcal{S}_{\alpha, \tau}^{*} g \in$ $W_{\tau}(Y)$, respectively, by that

$$
\begin{aligned}
\int_{0}^{T}\left\langle\left(\mathrm{D}_{0+}^{\alpha}-\mathcal{A}\right) \mathcal{S}_{\alpha, \tau} g, V\right\rangle_{X} \mathrm{~d} t & =\int_{0}^{T}\langle g, V\rangle_{X} \mathrm{~d} t, \\
\int_{0}^{T}\left\langle\left(\mathrm{D}_{T-}^{\alpha}-\mathcal{A}^{*}\right) \mathcal{S}_{\alpha, \tau}^{*} g, V\right\rangle_{X} \mathrm{~d} t & =\int_{0}^{T}\langle g, V\rangle_{X} \mathrm{~d} t,
\end{aligned}
$$

for all $V \in W_{\tau}(X)$. For any $g \in L^{1}\left(0, T ; X^{*}\right)$, define $\mathcal{S}_{1, \tau} g \in W_{\tau}(Y)$ and $\mathcal{S}_{1, \tau}^{*} g \in W_{\tau}(Y)$, respectively, by that

$$
\begin{aligned}
& \left(\left(\mathcal{S}_{1, \tau} g\right)(0+), V(0+)\right)_{Y}+\sum_{j=1}^{J-1}\left(\left(\mathcal{S}_{1, \tau} g\right)\left(t_{j}+\right)-\left(\mathcal{S}_{1, \tau} g\right)\left(t_{j}-\right), V\left(t_{j}+\right)\right)_{Y} \\
& -\int_{0}^{T}\left\langle\mathcal{A} \mathcal{S}_{1, \tau} g, V\right\rangle_{X} \mathrm{~d} t=\int_{0}^{T}\langle g, V\rangle_{X} \mathrm{~d} t, \\
& \left(\left(\mathcal{S}_{1, \tau}^{*} g\right)(T-), V(T-)\right)_{Y}+\sum_{j=1}^{J-1}\left(\left(\mathcal{S}_{1, \tau}^{*} g\right)\left(t_{j}-\right)-\left(\mathcal{S}_{1, \tau}^{*} g\right)\left(t_{j}+\right), V\left(t_{j}-\right)\right)_{Y} \\
& \quad-\int_{0}^{T}\left\langle\mathcal{A}^{*} \mathcal{S}_{1, \tau}^{*} g, V\right\rangle_{X} \mathrm{~d} t=\int_{0}^{T}\langle g, V\rangle_{X} \mathrm{~d} t,
\end{aligned}
$$

for all $V \in W_{\tau}(X)$. We will present some properties of $\mathcal{S}_{\alpha, \tau}, 0<\alpha \leqslant 1$, in Section 2.4. 
Remark 2.3. Scheme (48) is a famous discontinuous Galerkin method for parabolic equations (cf. [10]), and this scheme is a variant of the backward Euler difference scheme.

Remark 2.4. We note that the idea of using the Galerkin methods to discretize the time fractional calculus operators was firstly developed by McLean and Mustapha [45, 52, 53, 51]. The L1 scheme [39, 57] is widely used for the discretizations of the fractional diffusion equations. Jin et al. [24, Remark 3] discovered that the L1 scheme is equivalent to discretization (46) with uniform temporal grids. For the numerical analysis of discretization (46) with nonuniform temporal grids, we refer the reader to [36, 37].

Using the variational discretization concept proposed in [19], we consider the following temporally discrete problem:

$$
\min _{U \in U_{\mathrm{ad}}} J_{\alpha, \tau}(U):=\frac{1}{2}\left\|\left(\mathcal{S}_{\alpha, \tau} \mathcal{R}_{\theta_{0}} U\right)(T-)-y_{d}\right\|_{Y}^{2}+\frac{\nu}{2}\|U\|_{L^{2}(0, T ; Z)}^{2} .
$$

Note that (56) implies that $\left(\mathcal{S}_{\alpha, \tau} \mathcal{R}_{\theta_{0}} \cdot\right)(T-)$ is a bounded linear operator from $L^{2}(0, T ; Z)$ to $Y$. In addition, $U_{\text {ad }}$ is a convex, bounded and closed subset of $L^{2}(0, T ; Z)$. Hence, applying [60, Theorems 2.14 and 2.21] to problem (50) yields the following theorem, by Lemma 2.6.

Theorem 2.2. Problem (50) admits a unique solution $U \in U_{a d}$, and the following first-order optimality condition holds:

$$
\left\{\begin{array}{l}
Y=\mathcal{S}_{\alpha, \tau} \mathcal{R}_{\theta_{0}} U \\
P=\mathcal{S}_{\alpha, \tau}^{*}\left(\left(Y(T-)-y_{d}\right) \widehat{\delta}_{T}\right) \\
\int_{0}^{T}\left(\mathcal{R}_{\theta_{0}}^{*} P(t)+\nu U(t), V(t)-U(t)\right)_{Z} \mathrm{~d} t \geqslant 0 \quad \text { for all } V \in U_{a d},
\end{array}\right.
$$

where

$$
\widehat{\delta}_{T}:= \begin{cases}0 & \text { if } 0<t<T-\tau, \\ \tau^{-1} & \text { if } T-\tau<t<T .\end{cases}
$$

A simple modification of the proof of [38, Theorem 4.3] yields the following error estimate, by Lemma 2.7.

Theorem 2.3. Assume that $0<\alpha \leqslant 1$. Let $u$ and $y$ be defined in Theorem 2.1, and let $U$ and $Y$ be defined in Theorem 2.2. Then

$$
\begin{aligned}
& \|(y-Y)(T-)\|_{Y}+\sqrt{\nu}\|u-U\|_{L^{2}(0, T ; Z)} \\
\leqslant & C_{c_{0}, \omega_{0}, \mathcal{M}_{0}, T}\left(\left\|y_{d}\right\|_{Y}+\left\|\mathcal{R}_{\theta_{0}}\right\|_{\mathcal{L}\left(Z,\left[X^{*}, Y\right]_{\theta_{0}}\right)}\|u\|_{L^{\infty}(0, T ; Z)}\right) \times \\
& \left(1 /\left(\theta_{0} \alpha\right)+\sqrt{\varepsilon\left(\alpha, \theta_{0}, J\right)}+\varepsilon\left(\alpha, \theta_{0}, J\right) \tau^{\theta_{0} \alpha / 2}\right) \tau^{\theta_{0} \alpha / 2}
\end{aligned}
$$

where

$$
\varepsilon(\alpha, \theta, J):= \begin{cases}\frac{1}{\theta \alpha}+\frac{1-J^{\theta \alpha-1}}{1-\theta \alpha} & \text { if } \theta \alpha \neq 1 \\ \ln J & \text { if } \theta \alpha=1 .\end{cases}
$$




\subsection{Properties of $\mathcal{S}_{\alpha, \tau}$}

Assume that $0<\alpha \leqslant 1$ and $g \in L^{1}\left(0, T ; X^{*}\right)$. Define $\left\{W_{j}\right\}_{j=1}^{J} \subset Y$ as follows: for any $1 \leqslant k \leqslant J$,

$$
b_{1} W_{k}+\sum_{j=1}^{k-1}\left(b_{k-j+1}-2 b_{k-j}+b_{k-j-1}\right) W_{j}-\tau^{\alpha} \mathcal{A} W_{k}=\tau^{\alpha-1} \int_{t_{k-1}}^{t_{k}} g(t) \mathrm{d} t
$$

in $X^{*}$, where $b_{j}:=j^{1-\alpha} / \Gamma(2-\alpha)$ for each $0 \leqslant j \leqslant J$. A straightforward computation yields that (cf. [24, Remark 3])

$$
\left(\mathcal{S}_{\alpha, \tau} g\right)\left(t_{j}-\right)=W_{j} \quad \forall 1 \leqslant j \leqslant J .
$$

Hence, we conclude from (55) and Lemma 2.1 that, for any $0 \leqslant \beta \leqslant 1$,

$$
\mathcal{S}_{\alpha, \tau} \in \mathcal{L}\left(L^{1}\left(0, T ;\left[X^{*}, Y\right]_{1-\beta}\right), L^{\infty}\left(0, T ;[X, Y]_{\beta}\right)\right)
$$

and

$$
\lim _{\alpha \rightarrow 1-}\left\|\mathcal{S}_{\alpha, \tau}-\mathcal{S}_{1, \tau}\right\|_{\mathcal{L}\left(L^{1}\left(0, T ;\left[X^{*}, Y\right]_{1-\beta}\right), L^{\infty}\left(0, T ;[X, Y]_{\beta}\right)\right)}=0 .
$$

Symmetrically, for any $0 \leqslant \beta \leqslant 1$ we have that

$$
\mathcal{S}_{\alpha, \tau}^{*} \in \mathcal{L}\left(L^{1}\left(0, T ;\left[X^{*}, Y\right]_{1-\beta}\right), L^{\infty}\left(0, T ;[X, Y]_{\beta}\right)\right)
$$

and

$$
\lim _{\alpha \rightarrow 1-}\left\|\mathcal{S}_{\alpha, \tau}^{*}-\mathcal{S}_{1, \tau}^{*}\right\|_{\mathcal{L}\left(L^{1}\left(0, T ;\left[X^{*}, Y\right]_{1-\beta}\right), L^{\infty}\left(0, T ;[X, Y]_{\beta}\right)\right)}=0 .
$$

Lemma 2.6. Assume that $0<\alpha \leqslant 1$. For any $f \in L^{1}\left(0, T ; X^{*}\right)$ and $g \in$ $L^{1}(0, T ; Y)$,

$$
\int_{0}^{T}\left(\mathcal{S}_{\alpha, \tau} f, g\right)_{Y} \mathrm{~d} t=\int_{0}^{T}\left\langle f, \mathcal{S}_{\alpha, \tau}^{*} g\right\rangle_{X} \mathrm{~d} t .
$$

Proof. Assume that $0<\alpha<1$. By (58) we have $\mathcal{S}_{\alpha, \tau}^{*} g \in W_{\tau}(X)$, and then (47) and the density of $X$ in $Y$ yield that

$$
\int_{0}^{T}\left(\left(\mathrm{D}_{T-}^{\alpha}-\mathcal{A}^{*}\right) \mathcal{S}_{\alpha, \tau}^{*} g, V\right)_{Y} \mathrm{~d} t=\int_{0}^{T}(g, V)_{Y} \mathrm{~d} t
$$

for all $V \in W_{\tau}(Y)$. Hence,

$$
\begin{aligned}
\int_{0}^{T}\left(\mathcal{S}_{\alpha, \tau} f, g\right)_{Y} \mathrm{~d} t & =\int_{0}^{T}\left(\mathcal{S}_{\alpha, \tau} f,\left(\mathrm{D}_{T-}^{\alpha}-\mathcal{A}^{*}\right) \mathcal{S}_{\alpha, \tau}^{*} g\right)_{Y} \mathrm{~d} t \\
& =\int_{0}^{T}\left\langle\left(\mathrm{D}_{0+}^{\alpha}-\mathcal{A}\right) \mathcal{S}_{\alpha, \tau} f, \mathcal{S}_{\alpha, \tau}^{*} g\right\rangle_{X} \mathrm{~d} t \quad(\text { by }(4) \text { and }(6)) \\
& =\int_{0}^{T}\left\langle f, \mathcal{S}_{\alpha, \tau}^{*} g\right\rangle_{X} \mathrm{~d} t \quad(\text { by }(46)) .
\end{aligned}
$$

This proves (60) for $0<\alpha<1$. For the proof of (60) with $\alpha=1$, we refer the reader to [59, Chapter 12]. 
Lemma 2.7. Assume that $0<\alpha, \theta \leqslant 1$ and $p \in\{1, \infty\}$. For any $g \in$ $L^{p}\left(0, T ;\left[X^{*}, Y\right]_{\theta}\right)$ we have

$$
\left\|\left(\mathcal{S}_{\alpha}-\mathcal{S}_{\alpha, \tau}\right) g\right\|_{L^{p}(0, T ; Y)} \leqslant C_{c_{0}, \omega_{0}, \mathcal{M}_{0}} \varepsilon(\alpha, \theta, J) \tau^{\theta \alpha}\|g\|_{L^{p}\left(0, T ;\left[X^{*}, Y\right]_{\theta}\right)},
$$

and for any $v \in Y$ we have

$$
\left\|\mathcal{S}_{\alpha}\left(v \delta_{0}\right)-\mathcal{S}_{\alpha, \tau}\left(v \widehat{\delta}_{0}\right)\right\|_{L^{1}\left(0, T ;[X, Y]_{\theta}\right)} \leqslant C_{c_{0}, \omega_{0}, \mathcal{M}_{0}} \varepsilon(\alpha, \theta, J) \tau^{\theta \alpha}\|v\|_{Y},
$$

where $\varepsilon(\cdot, \cdot, \cdot)$ is defined by (54) and

$$
\widehat{\delta}_{0}(t):= \begin{cases}t_{1}^{-1} & \text { if } 0<t<t_{1}, \\ 0 & \text { if } t_{1}<t<T .\end{cases}
$$

The main task of the rest of this subsection is to prove Lemma 2.7. Firstly, we summarize some auxiliary results in [38]. Assume that $0<\alpha<1$. For any $z \in \mathbb{C} \backslash\{0\}$ with $-\pi \leqslant \Im z \leqslant \pi$, define

$$
\psi_{\alpha}(z):=\left(e^{z}-1\right)^{2} \sum_{k=-\infty}^{\infty}(z+2 k \pi i)^{\alpha-2} .
$$

There exists $\pi / 2<\omega^{*} \leqslant \omega_{0}$, depending only on $\omega_{0}$, such that

$$
e^{-z} \psi_{\alpha}(z) \in \Sigma_{\omega_{0}} \text { for all } z \in \Sigma_{\omega^{*}} \text { with }-\pi \leqslant \Im z \leqslant \pi
$$

and that, for any $z \in \Upsilon_{\omega^{*}} \backslash\{0\}$,

$$
\begin{gathered}
\left|e^{-z} \psi_{\alpha}(z)\right| \geqslant C_{\omega_{0}}|z|^{\alpha}, \\
\left|\psi_{\alpha}(z)-z^{\alpha}\right| \leqslant C_{\omega_{0}}|z|^{\alpha+1} .
\end{gathered}
$$

Define

$$
\mathcal{E}_{\alpha}(t):=\tau^{-1} \mathcal{E}_{\alpha,\lfloor t / \tau\rfloor}, \quad t>0,
$$

where $\lfloor\cdot\rfloor$ is the floor function and

$$
\mathcal{E}_{\alpha, j}:=\frac{1}{2 \pi i} \int_{\Upsilon_{\omega^{*}}} e^{j z} R\left(\tau^{-\alpha} e^{-z} \psi_{\alpha}(z), \mathcal{A}\right) \mathrm{d} z, \quad j \in \mathbb{N} .
$$

Following the proof of [38, Lemma 3.5], we obtain that, for any $g \in L^{1}(0, T ; Y)$,

$$
\left(\mathcal{S}_{\alpha, \tau} g\right)\left(t_{j}-\right)=\int_{0}^{t_{j}} \mathcal{E}_{\alpha}\left(t_{j}-t\right) g(t) \mathrm{d} t \quad \forall 1 \leqslant j \leqslant J .
$$

Since (11), (66), (67), (69) and (70) imply $\left\|\mathcal{E}_{\alpha}\right\|_{L^{\infty}\left(0, T ; \mathcal{L}\left(X^{*}, Y\right)\right)}<\infty$, from (56) and the fact that $L^{1}(0, T ; Y)$ is dense in $L^{1}\left(0, T ; X^{*}\right)$ we conclude that (71) holds for all $g \in L^{1}\left(0, T ; X^{*}\right)$.

Secondly, we present some auxiliary estimates in the following three lemmas.

Lemma 2.8. For any $0<\alpha<1,0 \leqslant \theta \leqslant 1$ and $z \in \Upsilon_{\omega^{*}} \backslash\{0\}$,

$$
\begin{aligned}
\left\|e^{z} R\left(\tau^{-\alpha} z^{\alpha}, \mathcal{A}\right)-R\left(\tau^{-\alpha} e^{-z} \psi_{\alpha}(z), \mathcal{A}\right)\right\|_{\mathcal{L}\left(Y,[X, Y]_{\theta}\right)} & \leqslant \frac{C_{c_{0}, \omega_{0}, \mathcal{M}_{0}}|z|}{1+\left(\tau^{-\alpha}|z|^{\alpha}\right)^{\theta}}, \\
\left\|e^{z} R\left(\tau^{-\alpha} z^{\alpha}, \mathcal{A}\right)-R\left(\tau^{-\alpha} e^{-z} \psi_{\alpha}(z), \mathcal{A}\right)\right\|_{\mathcal{L}\left(\left[X^{*}, Y\right]_{\theta}, Y\right)} & \leqslant \frac{C_{c_{0}, \omega_{0}, \mathcal{M}_{0}|z|}}{1+\left(\tau^{-\alpha}|z|^{\alpha}\right)^{\theta}} .
\end{aligned}
$$


Proof. A straightforward computation gives

$$
\begin{aligned}
& e^{z} R\left(\tau^{-\alpha} z^{\alpha}, \mathcal{A}\right)-R\left(\tau^{-\alpha} e^{-z} \psi_{\alpha}(z), \mathcal{A}\right) \\
= & \left(\tau^{-\alpha}\left(\psi_{\alpha}(z)-z^{\alpha}\right)+\left(1-e^{z}\right) \mathcal{A}\right) R\left(\tau^{-\alpha} z^{\alpha}, \mathcal{A}\right) R\left(\tau^{-\alpha} e^{-z} \psi_{\alpha}(z), \mathcal{A}\right) \\
= & \mathbb{I}_{1}+\mathbb{I}_{2},
\end{aligned}
$$

where

$$
\begin{aligned}
& \mathbb{I}_{1}:=\tau^{-\alpha}\left(\psi_{\alpha}(z)-z^{\alpha}\right) R\left(\tau^{-\alpha} z^{\alpha}, \mathcal{A}\right) R\left(\tau^{-\alpha} e^{-z} \psi_{\alpha}(z), \mathcal{A}\right) \\
& \mathbb{I}_{2}:=\left(1-e^{z}\right) \mathcal{A} R\left(\tau^{-\alpha} z^{\alpha}, \mathcal{A}\right) R\left(\tau^{-\alpha} e^{-z} \psi_{\alpha}(z), \mathcal{A}\right)
\end{aligned}
$$

We conclude from (9), (66) and (67) that, for any $0 \leqslant \beta \leqslant 1$,

$$
\begin{aligned}
\left\|R\left(\tau^{-\alpha} z^{\alpha}, \mathcal{A}\right)\right\|_{\mathcal{L}\left(Y,[X, Y]_{\beta}\right)} & \leqslant C_{\mathcal{M}_{0}}\left(1+\left(\tau^{-\alpha}|z|^{\alpha}\right)^{\beta}\right)^{-1} \\
\left\|R\left(\tau^{-\alpha} e^{-z} \psi_{\alpha}(z), \mathcal{A}\right)\right\|_{\mathcal{L}\left(Y,[X, Y]_{\beta}\right)} & \leqslant C_{c_{0}, \omega_{0}, \mathcal{M}_{0}}\left(1+\left(\tau^{-\alpha}|z|^{\alpha}\right)^{\beta}\right)^{-1} .
\end{aligned}
$$

For $\mathbb{I}_{1}$ we have, by (68), (74) and (75),

$$
\begin{aligned}
& \left\|\mathbb{I}_{1}\right\|_{\mathcal{L}\left(Y,[X, Y]_{\theta}\right)} \\
\leqslant & C_{\omega_{0}} \tau^{-\alpha}|z|^{\alpha+1}\left\|R\left(\tau^{-\alpha} z^{\alpha}, \mathcal{A}\right)\right\|_{\mathcal{L}\left(Y,[X, Y]_{\theta}\right)}\left\|R\left(\tau^{-\alpha} e^{-z} \psi_{\alpha}(z), \mathcal{A}\right)\right\|_{\mathcal{L}(Y)} \\
\leqslant & \frac{C_{c_{0}, \omega_{0}, \mathcal{M}_{0}} \tau^{-\alpha}|z|^{\alpha+1}}{\left(1+\tau^{-\alpha}|z|^{\alpha}\right)\left(1+\left(\tau^{-\alpha}|z|^{\alpha}\right)^{\theta}\right)} \leqslant \frac{C_{c_{0}, \omega_{0}, \mathcal{M}_{0}}|z|}{1+\left(\tau^{-\alpha}|z|^{\alpha}\right)^{\theta}}
\end{aligned}
$$

Since

$$
\begin{aligned}
& \left\|\mathcal{A} R\left(\tau^{-\alpha} z^{\alpha}, \mathcal{A}\right) R\left(\tau^{-\alpha} e^{-z} \psi_{\alpha}(z), \mathcal{A}\right)\right\|_{\mathcal{L}\left(Y,[X, Y]_{\theta}\right)} \\
= & \left\|\left(\tau^{-\alpha} z^{\alpha} R\left(\tau^{-\alpha} z^{\alpha}, \mathcal{A}\right)-I\right) R\left(\tau^{-\alpha} e^{-z} \psi_{\alpha}(z), \mathcal{A}\right)\right\|_{\mathcal{L}\left(Y,[X, Y]_{\theta}\right)} \\
\leqslant & \left\|\tau^{-\alpha} z^{\alpha} R\left(\tau^{-\alpha} z^{\alpha}, \mathcal{A}\right)\right\|_{\mathcal{L}\left(Y,[X, Y]_{\theta}\right)}\left\|R\left(\tau^{-\alpha} e^{-z} \psi_{\alpha}(z), \mathcal{A}\right)\right\|_{\mathcal{L}(Y)} \\
& \quad+\left\|R\left(\tau^{-\alpha} e^{-z} \psi_{\alpha}(z), \mathcal{A}\right)\right\|_{\left(Y,[X, Y]_{\theta}\right)} \\
\leqslant & \frac{C_{c_{0}, \omega_{0}, \mathcal{M}_{0}}}{1+\left(\tau^{-\alpha}|z|^{\alpha}\right)^{\theta}} \quad(\text { by }(74) \text { and }(75)),
\end{aligned}
$$

we obtain

$$
\left\|\mathbb{I}_{2}\right\|_{\mathcal{L}\left(Y,[X, Y]_{\theta}\right)} \leqslant \frac{C_{c_{0}, \omega_{0}, \mathcal{M}_{0}}|z|}{1+\left(\tau^{-\alpha}|z|^{\alpha}\right)^{\theta}} .
$$

Combining the above estimates of $\mathbb{I}_{1}$ and $\mathbb{I}_{2}$ proves (72). Since (73) can be derived analogously, this completes the proof.

Lemma 2.9. Assume that $0<\alpha<1$ and $0 \leqslant \theta \leqslant 1$. Then

$$
\begin{aligned}
& \max _{1 \leqslant j \leqslant J} j^{2-\theta \alpha}\left\|E_{\alpha}\left(t_{j}\right)-\mathcal{E}_{\alpha}\left(t_{j}-\right)\right\|_{\mathcal{L}\left(Y,[X, Y]_{\theta}\right)} \leqslant C_{c_{0}, \omega_{0}, \mathcal{M}_{0}} \tau^{\theta \alpha-1}, \\
& \max _{1 \leqslant j \leqslant J} j^{2-\theta \alpha}\left\|E_{\alpha}\left(t_{j}\right)-\mathcal{E}_{\alpha}\left(t_{j}-\right)\right\|_{\mathcal{L}\left(\left[X^{*}, Y\right]_{\theta}, Y\right)} \leqslant C_{c_{0}, \omega_{0}, \mathcal{M}_{0}} \tau^{\theta \alpha-1} .
\end{aligned}
$$

Proof. For each $1 \leqslant j \leqslant J$, inserting $t=t_{j}$ into (17) yields

$$
E_{\alpha}\left(t_{j}\right)=\frac{1}{2 \pi i} \int_{\Gamma_{\omega^{*}}} e^{t_{j} z} R\left(z^{\alpha}, \mathcal{A}\right) \mathrm{d} z=\frac{\tau^{-1}}{2 \pi i} \int_{\Gamma_{\omega^{*}}} e^{j z} R\left(\tau^{-\alpha} z^{\alpha}, \mathcal{A}\right) \mathrm{d} z,
$$


and so we conclude from (69) and (70) that

$$
E_{\alpha}\left(t_{j}\right)-\mathcal{E}_{\alpha}\left(t_{j}-\right)=\mathbb{I}_{1}+\mathbb{I}_{2},
$$

where

$$
\begin{aligned}
& \mathbb{I}_{1}:=\frac{\tau^{-1}}{2 \pi i} \int_{\Gamma_{\omega^{*}} \backslash \Upsilon_{\omega^{*}}} e^{j z} R\left(\tau^{-\alpha} z^{\alpha}, \mathcal{A}\right) \mathrm{d} z \\
& \mathbb{I}_{2}:=\frac{\tau^{-1}}{2 \pi i} \int_{\Upsilon_{\omega^{*}}} e^{(j-1) z}\left(e^{z} R\left(\tau^{-\alpha} z^{\alpha}, \mathcal{A}\right)-R\left(\tau^{-\alpha} e^{-z} \psi_{\alpha}(z), \mathcal{A}\right)\right) \mathrm{d} z .
\end{aligned}
$$

A straightforward computation gives

$$
\begin{aligned}
& \left\|\mathbb{I}_{1}\right\|_{\mathcal{L}\left(Y,[X, Y]_{\theta}\right)} \\
\leqslant & \left.C_{c_{0}, \mathcal{M}_{0}} \tau^{-1} \int_{\pi / \sin \omega^{*}}^{\infty} e^{j \cos \omega^{*} r}\left(1+\left(\tau^{-\alpha} r^{\alpha}\right)^{\theta}\right)^{-1} \mathrm{~d} r \quad \text { (by }(9)\right) \\
< & C_{c_{0}, \mathcal{M}_{0}} \tau^{\theta \alpha-1} \int_{\pi / \sin \omega^{*}}^{\infty} e^{j \cos \omega^{*} r} r^{-\theta \alpha} \mathrm{d} r \\
< & C_{c_{0}, \mathcal{M}_{0}} \tau^{\theta \alpha-1} \int_{\pi / \sin \omega^{*}}^{\infty} e^{j \cos \omega^{*} r} \mathrm{~d} r \\
< & C_{c_{0}, \omega_{0}, \mathcal{M}_{0}} \tau^{\theta \alpha-1} j^{-1} e^{j \pi \cot \omega^{*}}
\end{aligned}
$$

and

$$
\begin{aligned}
& \left\|\mathbb{I}_{2}\right\|_{\mathcal{L}\left(Y,[X, Y]_{\theta}\right)} \\
< & C_{c_{0}, \omega_{0}, \mathcal{M}_{0}} \tau^{-1} \int_{0}^{\pi / \sin \omega^{*}} e^{(j-1) \cos \omega^{*} r} r\left(1+\left(\tau^{-\alpha} r^{\alpha}\right)^{\theta}\right)^{-1} \mathrm{~d} r \quad(\text { by }(72)) \\
< & C_{c_{0}, \omega_{0}, \mathcal{M}_{0}} \tau^{\theta \alpha-1} \int_{0}^{\pi / \sin \omega^{*}} e^{(j-1) \cos \omega^{*} r} r^{1-\theta \alpha} \mathrm{d} r \\
< & C_{c_{0}, \omega_{0}, \mathcal{M}_{0}} \tau^{\theta \alpha-1} j^{\theta \alpha-2} .
\end{aligned}
$$

Together the above estimates of $\mathbb{I}_{1}$ and $\mathbb{I}_{2}$ yields (76). Since (77) can be proved analogously by (11) and (73), this completes the proof.

Lemma 2.10. For any $0<\alpha<1$ and $0<\theta \leqslant 1$,

$$
\begin{aligned}
\left\|E_{\alpha}-\mathcal{E}_{\alpha}\right\|_{L^{1}\left(0, T ; \mathcal{L}\left(Y,[X, Y]_{\theta}\right)\right)} & \leqslant C_{c_{0}, \omega_{0}, \mathcal{M}_{0}}\left(\frac{1}{\theta \alpha}+\frac{1-J^{\theta \alpha-1}}{1-\theta \alpha}\right) \tau^{\theta \alpha}, \\
\left\|E_{\alpha}-\mathcal{E}_{\alpha}\right\|_{L^{1}\left(0, T ; \mathcal{L}\left(\left[X^{*}, Y\right]_{\theta}, Y\right)\right)} & \leqslant C_{c_{0}, \omega_{0}, \mathcal{M}_{0}}\left(\frac{1}{\theta \alpha}+\frac{1-J^{\theta \alpha-1}}{1-\theta \alpha}\right) \tau^{\theta \alpha} .
\end{aligned}
$$

Proof. By (20) we have

$$
\left\|E_{\alpha}-E_{\alpha}\left(t_{1}\right)\right\|_{L^{1}\left(0, t_{1} ; \mathcal{L}\left(Y,[X, Y]_{\theta}\right)\right)}<C_{c_{0}, \omega_{0}, \mathcal{M}_{0}} \tau^{\theta \alpha} /(\theta \alpha),
$$

and a straightforward calculation gives

$$
\begin{aligned}
& \sum_{j=2}^{J}\left\|E_{\alpha}-E_{\alpha}\left(t_{j}\right)\right\|_{L^{1}\left(t_{j-1}, t_{j} ; \mathcal{L}\left(Y,[X, Y]_{\theta}\right)\right)} \\
\leqslant & \tau\left\|E_{\alpha}^{\prime}\right\|_{L^{1}\left(t_{1}, T ; \mathcal{L}\left(Y,[X, Y]_{\theta}\right)\right)} \leqslant C_{c_{0}, \omega_{0}, \mathcal{M}_{0}} \tau \int_{t_{1}}^{T} t^{\theta \alpha-2} \mathrm{~d} t \quad(\text { by }(22)) \\
= & C_{c_{0}, \omega_{0}, \mathcal{M}_{0}} \tau^{\theta \alpha}\left(1-J^{\theta \alpha-1}\right) /(1-\theta \alpha) .
\end{aligned}
$$


It follows that

$$
\begin{aligned}
& \sum_{j=1}^{J}\left\|E_{\alpha}-E_{\alpha}\left(t_{j}\right)\right\|_{L^{1}\left(t_{j-1}, t_{j} ; \mathcal{L}\left(Y,[X, Y]_{\theta}\right)\right)} \\
\leqslant & C_{c_{0}, \omega_{0}, \mathcal{M}_{0}}\left(\frac{1}{\theta \alpha}+\frac{1-J^{\theta \alpha-1}}{1-\theta \alpha}\right) \tau^{\theta \alpha} .
\end{aligned}
$$

In addition, by (76),

$$
\begin{aligned}
\sum_{j=1}^{J} \tau\left\|E_{\alpha}\left(t_{j}\right)-\mathcal{E}_{\alpha}\left(t_{j}-\right)\right\|_{\mathcal{L}\left(Y,[X, Y]_{\theta}\right)} & \leqslant C_{c_{0}, \omega_{0}, \mathcal{M}_{0}} \tau^{\theta \alpha} \sum_{j=1}^{J} j^{\theta \alpha-2} \\
& \leqslant C_{c_{0}, \omega_{0}, \mathcal{M}_{0}} \tau^{\theta \alpha}\left(1-J^{\theta \alpha-1}\right) /(1-\theta \alpha) .
\end{aligned}
$$

Consequently,

$$
\begin{aligned}
& \left\|E_{\alpha}-\mathcal{E}_{\alpha}\right\|_{L^{1}\left(0, T ; \mathcal{L}\left(Y,[X, Y]_{\theta}\right)\right)} \\
\leqslant & \sum_{j=1}^{J}\left(\left\|E_{\alpha}-E_{\alpha}\left(t_{j}\right)\right\|_{L^{1}\left(t_{j-1}, t_{j} ; \mathcal{L}\left(Y,[X, Y]_{\theta}\right)\right)}+\right. \\
& \left.\tau\left\|E_{\alpha}\left(t_{j}\right)-\mathcal{E}_{\alpha}\left(t_{j}-\right)\right\|_{L^{1}\left(t_{j-1}, t_{j} ; \mathcal{L}\left(Y,[X, Y]_{\theta}\right)\right)}\right) \\
\leqslant & C_{c_{0}, \omega_{0}, \mathcal{M}_{0}}\left(\frac{1}{\theta \alpha}+\frac{1-J^{\theta \alpha-1}}{1-\theta \alpha}\right) \tau^{\theta \alpha},
\end{aligned}
$$

which proves (78). Since (79) can be proved analogously by (21), (23) and (77), this completes the proof.

Thirdly, we prove that (62) holds for $0<\alpha<1$ and $p=1$.

Lemma 2.11. Assume that $0<\alpha<1$ and $0<\theta \leqslant 1$. For any $g \in$ $L^{1}\left(0, T ;\left[X^{*}, Y\right]_{\theta}\right)$, we have

$$
\left\|\left(\mathcal{S}_{\alpha}-\mathcal{S}_{\alpha, \tau}\right) g\right\|_{L^{1}(0, T ; Y)} \leqslant C_{c_{0}, \omega_{0}, \mathcal{M}_{0}}\left(\frac{1}{\theta \alpha}+\frac{1-J^{\theta \alpha-1}}{1-\theta \alpha}\right) \tau^{\theta \alpha}\|g\|_{L^{1}\left(0, T ;\left[X^{*}, Y\right]_{\theta}\right)} .
$$

Proof. Step 1. Let us prove

$$
\begin{aligned}
& \int_{0}^{T}\left\|\int_{0}^{t}\left(E_{\alpha}(t-s)-E_{\alpha}(t-s+\tau)\right) g(s) \mathrm{d} s\right\|_{Y} \mathrm{~d} t \\
\leqslant & C_{c_{0}, \omega_{0}, \mathcal{M}_{0}}\left(\frac{1}{\theta \alpha}+\frac{1-J^{\theta \alpha-1}}{1-\theta \alpha}\right) \tau^{\theta \alpha}\|g\|_{L^{1}\left(0, T ;\left[X^{*}, Y\right]_{\theta}\right)} .
\end{aligned}
$$

A straightforward computation gives

$$
\int_{0}^{\tau}\left\|E_{\alpha}(t)-E_{\alpha}(t+\tau)\right\|_{\mathcal{L}\left(\left[X^{*}, Y\right]_{\theta}, Y\right)} \mathrm{d} t \leqslant C_{c_{0}, \omega_{0}, \mathcal{M}_{0}} \tau^{\theta \alpha} /(\theta \alpha) \quad(\text { by }(21))
$$

and

$$
\begin{aligned}
& \int_{\tau}^{T}\left\|E_{\alpha}(t)-E_{\alpha}(t+\tau)\right\|_{\mathcal{L}\left(\left[X^{*}, Y\right]_{\theta}, Y\right)} \mathrm{d} t \\
\leqslant & C_{c_{0}, \omega_{0}, \mathcal{M}_{0}} \tau \int_{\tau}^{T} t^{\theta \alpha-2} \mathrm{~d} t \quad(\text { by }(23)) \\
\leqslant & C_{c_{0}, \omega_{0}, \mathcal{M}_{0}} \tau^{\theta \alpha}\left(1-J^{\theta \alpha-1}\right) /(1-\theta \alpha) .
\end{aligned}
$$


It follows that

$$
\int_{0}^{T}\left\|E_{\alpha}(t)-E_{\alpha}(t+\tau)\right\|_{\mathcal{L}\left(\left[X^{*}, Y\right]_{\theta}, Y\right)} \leqslant C_{c_{0}, \omega_{0}, \mathcal{M}_{0}}\left(\frac{1}{\theta \alpha}+\frac{1-J^{\theta \alpha-1}}{1-\theta \alpha}\right) \tau^{\theta \alpha} .
$$

Hence, (83) follows from the estimate

$$
\begin{aligned}
& \int_{0}^{T}\left\|\int_{0}^{t}\left(E_{\alpha}(t-s)-E_{\alpha}(t-s+\tau)\right) g(s) \mathrm{d} s\right\|_{Y} \mathrm{~d} t \\
\leqslant & \int_{0}^{T} \int_{0}^{t}\left\|E_{\alpha}(t-s)-E_{\alpha}(t-s+\tau)\right\|_{\mathcal{L}\left(\left[X^{*}, Y\right]_{\theta}, Y\right)}\|g(s)\|_{\left[X^{*}, Y\right]_{\theta}} \mathrm{d} s \mathrm{~d} t \\
= & \int_{0}^{T} \int_{s}^{T}\left\|E_{\alpha}(t-s)-E_{\alpha}(t-s+\tau)\right\|_{\mathcal{L}\left(\left[X^{*}, Y\right]_{\theta}, Y\right)}\|g(s)\|_{\left[X^{*}, Y\right]_{\theta}} \mathrm{d} t \mathrm{~d} s \\
= & \int_{0}^{T} \int_{0}^{T-s}\left\|E_{\alpha}(r)-E_{\alpha}(r+\tau)\right\|_{\mathcal{L}\left(\left[X^{*}, Y\right]_{\theta}, Y\right)} \mathrm{d} r\|g(s)\|_{\left[X^{*}, Y\right]_{\theta}} \mathrm{d} s \\
\leqslant & \|g\|_{L^{1}\left(0, T ;\left[X^{*}, Y\right]_{\theta}\right)} \int_{0}^{T}\left\|E_{\alpha}(r)-E_{\alpha}(r+\tau)\right\|_{\mathcal{L}\left(\left[X^{*}, Y\right]_{\theta}, Y\right)} \mathrm{d} s .
\end{aligned}
$$

Step 2. Let us prove that

$\int_{0}^{T}\left\|\int_{0}^{t} E_{\alpha}(t-s+\tau) g(s)-G(t)\right\|_{Y} \mathrm{~d} t \leqslant C_{c_{0}, \omega_{0}, \mathcal{M}_{0}} \frac{1-J^{\theta \alpha-1}}{1-\theta \alpha} \tau^{\theta \alpha}\|g\|_{L^{1}\left(0, T ;\left[X^{*}, Y\right]_{\theta}\right)}$,

where $G \in W_{\tau}(Y)$ is defined by

$$
G\left(t_{j}-\right):=\sum_{k=1}^{j} E_{\alpha}\left(t_{j}-t_{k}+\tau\right) \int_{t_{k-1}}^{t_{k}} g(s) \mathrm{d} s, \quad 1 \leqslant j \leqslant J
$$

For any $t_{j-1}<t<t_{j}$ with $1 \leqslant j \leqslant J$, by (21) we have

$$
\begin{aligned}
& \left\|\int_{t_{j-1}}^{t} E_{\alpha}(t-s+\tau) g(s) \mathrm{d} s-E_{\alpha}(\tau) \int_{t_{j-1}}^{t_{j}} g(s) \mathrm{d} s\right\|_{Y} \\
\leqslant & C_{c_{0}, \omega_{0}, \mathcal{M}_{0}} \tau^{\theta \alpha-1}\|g\|_{L^{1}\left(t_{j-1}, t_{j} ;\left[X^{*}, Y\right]_{\theta}\right)},
\end{aligned}
$$

and by (23) we have

$$
\begin{aligned}
& \left\|\sum_{k=1}^{j-1} \int_{t_{k-1}}^{t_{k}}\left(E_{\alpha}(t-s+\tau)-E_{\alpha}\left(t_{j}-t_{k}+\tau\right)\right) g(s) \mathrm{d} s\right\|_{Y} \\
\leqslant & C_{c_{0}, \omega_{0}, \mathcal{M}_{0}} \sum_{k=1}^{j-1} \tau\left(t_{j}-t_{k}+\tau\right)^{\theta \alpha-2}\|g\|_{L^{1}\left(t_{k-1}, t_{k} ;\left[X^{*}, Y\right]_{\theta}\right)} \\
= & C_{c_{0}, \omega_{0}, \mathcal{M}_{0}} \tau^{\theta \alpha-1} \sum_{k=1}^{j-1}(j-k+1)^{\theta \alpha-2}\|g\|_{L^{1}\left(t_{k-1}, t_{k} ;\left[X^{*}, Y\right]_{\theta}\right)} .
\end{aligned}
$$


Hence, for each $t_{j-1}<t<t_{j}$ with $1 \leqslant j \leqslant J$,

$$
\begin{aligned}
& \left\|\int_{0}^{t} E_{\alpha}(t-s+\tau) g(s) \mathrm{d} s-G\left(t_{j}-\right)\right\|_{Y} \\
\leqslant & \left\|\int_{t_{j-1}}^{t} E_{\alpha}(t-s+\tau) g(s) \mathrm{d} s-E_{\alpha}(\tau) \int_{t_{j-1}}^{t_{j}} g(s) \mathrm{d} s\right\|_{Y}+ \\
& \quad\left\|\sum_{k=1}^{j-1} \int_{t_{k-1}}^{t_{k}}\left(E_{\alpha}(t-s+\tau)-E_{\alpha}\left(t_{j}-t_{k}+\tau\right)\right) g(s) \mathrm{d} s\right\|_{Y} \\
\leqslant & C_{c_{0}, \omega_{0}, \mathcal{M}_{0}} \tau^{\theta \alpha-1} \sum_{k=1}^{j}(j-k+1)^{\theta \alpha-2}\|g\|_{L^{1}\left(t_{k-1}, t_{k} ;\left[X^{*}, Y\right]_{\theta}\right)} .
\end{aligned}
$$

It follows that, for each $1 \leqslant j \leqslant J$,

$$
\begin{aligned}
& \int_{t_{j-1}}^{t_{j}}\left\|\int_{0}^{t} E_{\alpha}(t+\tau-s) g(s) \mathrm{d} s-G(t)\right\|_{Y} \mathrm{~d} t \\
\leqslant & C_{c_{0}, \omega_{0}, \mathcal{M}_{0}} \tau^{\theta \alpha} \sum_{k=1}^{j}(j-k+1)^{\theta \alpha-2}\|g\|_{L^{1}\left(t_{k-1}, t_{k} ;\left[X^{*}, Y\right]_{\theta}\right)} .
\end{aligned}
$$

Therefore,

$$
\begin{aligned}
& \int_{0}^{T}\left\|\int_{0}^{t} E_{\alpha}(t+\tau-s) g(s) \mathrm{d} s-G(t)\right\|_{Y} \mathrm{~d} t \\
\leqslant & C_{c_{0}, \omega_{0}, \mathcal{M}_{0}} \tau^{\theta \alpha} \sum_{j=1}^{J} \sum_{k=1}^{j}(j-k+1)^{\theta \alpha-2}\|g\|_{L^{1}\left(t_{k-1}, t_{k} ;\left[X^{*}, Y\right]_{\theta}\right)} \\
= & C_{c_{0}, \omega_{0}, \mathcal{M}_{0}} \tau^{\theta \alpha} \sum_{k=1}^{J} \sum_{j=k}^{J}(j-k+1)^{\theta \alpha-2}\|g\|_{L^{1}\left(t_{k-1}, t_{k} ;\left[X^{*}, Y\right]_{\theta}\right)} \\
\leqslant & C_{c_{0}, \omega_{0}, \mathcal{M}_{0}} \tau^{\theta \alpha}\|g\|_{L^{1}\left(0, T ;\left[X^{*}, Y\right]_{\theta}\right)} \sum_{m=1}^{J} m^{\theta \alpha-2} .
\end{aligned}
$$

The desired estimate (84) then follows from the simple inequality

$$
\sum_{m=1}^{J} m^{\theta \alpha-2}<1+\frac{1-J^{\theta \alpha-1}}{1-\theta \alpha} .
$$

Step 3. Let us prove that

$$
\int_{0}^{T}\left\|\left(\mathcal{S}_{\alpha, \tau} g\right)(t)-G(t)\right\|_{Y} \mathrm{~d} t \leqslant C_{c_{0}, \omega_{0}, \mathcal{M}_{0}} \frac{1-J^{\theta \alpha-1}}{1-\theta \alpha} \tau^{\theta \alpha}\|g\|_{L^{1}\left(0, T ;\left[X^{*}, Y\right]_{\theta}\right)} .
$$


Noting that $\mathcal{S}_{\alpha, \tau} g$ and $G$ are piecewise constant, we have

$$
\begin{aligned}
& \int_{0}^{T}\left\|\left(\mathcal{S}_{\alpha, \tau} g\right)(t)-G(t)\right\|_{Y} \mathrm{~d} t \\
= & \sum_{j=1}^{J} \tau\left\|\left(\mathcal{S}_{\alpha, \tau} g\right)\left(t_{j}-\right)-G\left(t_{j}-\right)\right\|_{Y} \\
\leqslant & \sum_{j=1}^{J} \tau\left\|\sum_{k=1}^{j}\left(\mathcal{E}_{\alpha}\left(\left(t_{j}-t_{k}+\tau\right)-\right)-E_{\alpha}\left(t_{j}-t_{k}+\tau\right)\right) \int_{t_{k-1}}^{t_{k}} g(s) \mathrm{d} s\right\|_{Y}(\text { by }(71) \text { and }(85)) \\
\leqslant & \sum_{j=1}^{J} \tau \sum_{k=1}^{j}\left\|\mathcal{E}_{\alpha}\left(\left(t_{j}-t_{k}+\tau\right)-\right)-E_{\alpha}\left(t_{j}-t_{k}+\tau\right)\right\|_{\mathcal{L}\left(\left[X^{*}, Y\right]_{\theta}, Y\right)} \int_{t_{k-1}}^{t_{k}}\|g(s)\|_{\left[X^{*}, Y\right]_{\theta}} \mathrm{d} s \\
= & \tau \sum_{k=1}^{J} \sum_{j=k}^{J}\left\|\mathcal{E}_{\alpha}\left(\left(t_{j}-t_{k}+\tau\right)-\right)-E_{\alpha}\left(t_{j}-t_{k}+\tau\right)\right\|_{\mathcal{L}\left(\left[X^{*}, Y\right]_{\theta}, Y\right)} \int_{t_{k-1}}^{t_{k}}\|g(s)\|_{\left[X^{*}, Y\right]_{\theta}} \mathrm{d} s \\
\leqslant & \tau\|g\|_{L^{1}\left(0, T ;\left[X^{*}, Y\right]_{\theta}\right)} \sum_{m=1}^{J}\left\|\mathcal{E}_{\alpha}\left(t_{m}-\right)-E\left(t_{m}\right)\right\|_{\mathcal{L}\left(\left[X^{*}, Y\right]_{\theta}, Y\right)} \\
\leqslant & C_{c_{0}, \omega_{0}, \mathcal{M}_{0}} \tau^{\theta \alpha}\|g\|_{L^{1}\left(0, T ;\left[X^{*}, Y\right]_{\theta}\right)} \sum_{m=1}^{J} m^{\theta \alpha-2} \quad(\text { by }(77)) .
\end{aligned}
$$

Hence, (87) follows from (86).

Step 4. By (26) we have

$$
\begin{aligned}
& \left\|\left(\mathcal{S}_{\alpha}-\mathcal{S}_{\alpha, \tau}\right) g\right\|_{L^{1}(0, T ; Y)} \\
& \leqslant \int_{0}^{T}\left\|\int_{0}^{t}\left(E_{\alpha}(t-s)-E_{\alpha}(t-s+\tau)\right) g(s) \mathrm{d} s\right\|_{Y} \mathrm{~d} t \\
& \quad+\int_{0}^{T}\left\|\int_{0}^{t} E_{\alpha}(t-s+\tau) g(s) \mathrm{d} s-G(t)\right\|_{Y} \mathrm{~d} t \\
& \quad+\int_{0}^{T}\left\|\left(\mathcal{S}_{\alpha, \tau} g\right)(t)-G(t)\right\|_{Y} \mathrm{~d} t .
\end{aligned}
$$

Therefore, combining (83), (84) and (87) proves (82) and thus concludes the proof.

Fourthly, let us prove that (62) holds for $0<\alpha<1$ and $p=\infty$.

Lemma 2.12. Assume that $0<\alpha<1$ and $0<\theta \leqslant 1$. For any $g \in$ $L^{\infty}\left(0, T ;\left[X^{*}, Y\right]_{\theta}\right)$ we have

$$
\begin{aligned}
& \left\|\left(\mathcal{S}_{\alpha}-\mathcal{S}_{\alpha, \tau}\right) g\right\|_{L^{\infty}(0, T ; Y)} \\
\leqslant & C_{c_{0}, \omega_{0}, \mathcal{M}_{0}}\left(\frac{1}{\theta \alpha}+\frac{1-J^{\theta \alpha-1}}{1-\theta \alpha}\right) \tau^{\theta \alpha}\|g\|_{L^{\infty}\left(0, T ;\left[X^{*}, Y\right]_{\theta}\right)} .
\end{aligned}
$$

Proof. By virtue of (26) and (71) we have that

$$
\begin{aligned}
& \max _{1 \leqslant j \leqslant J}\left\|\left(\mathcal{S}_{\alpha} g\right)\left(t_{j}\right)-\left(\mathcal{S}_{\alpha, \tau} g\right)\left(t_{j}-\right)\right\|_{Y} \\
\leqslant & \left\|E_{\alpha}-\mathcal{E}_{\alpha}\right\|_{L^{1}\left(0, T ; \mathcal{L}\left(\left[X^{*}, Y\right]_{\theta}, Y\right)\right)}\|g\|_{L^{\infty}\left(0, T ;\left[X^{*}, Y\right]_{\theta}\right)},
\end{aligned}
$$


so that (79) implies that

$$
\begin{aligned}
& \max _{1 \leqslant j \leqslant J}\left\|\left(\mathcal{S}_{\alpha} g\right)\left(t_{j}\right)-\left(\mathcal{S}_{\alpha, \tau} g\right)\left(t_{j}-\right)\right\|_{Y} \\
\leqslant & C_{c_{0}, \omega_{0}, \mathcal{M}_{0}}\left(\frac{1}{\theta \alpha}+\frac{1-J^{\theta \alpha-1}}{1-\theta \alpha}\right)\|g\|_{L^{\infty}\left(0, T ;\left[X^{*}, Y\right]_{\theta}\right)} .
\end{aligned}
$$

It remains therefore to prove that

$$
\begin{aligned}
& \max _{1 \leqslant j \leqslant J}\left\|\mathcal{S}_{\alpha} g-\left(\mathcal{S}_{\alpha} g\right)\left(t_{j}\right)\right\|_{L^{\infty}\left(t_{j-1}, t_{j} ; Y\right)} \\
\leqslant & C_{c_{0}, \omega_{0}, \mathcal{M}_{0}}\left(\frac{1}{\theta \alpha}+\frac{1-J^{\theta \alpha-1}}{1-\theta \alpha}\right) \tau^{\theta \alpha}\|g\|_{L^{\infty}\left(0, T ;\left[X^{*}, Y\right]_{\theta}\right)} .
\end{aligned}
$$

But this is easily derived by (21), (23) and (26); see the proof of [9, Thoerem $2.6]$ for the relevant techniques. This completes the proof.

Finally, we are in a position to conclude the proof of Lemma 2.7 as follows.

Proof of Lemma 2.7. Let us first prove (62). By Lemmas 2.11 and 2.12 we have that (62) holds for all $0<\alpha<1$ and $p \in\{1, \infty\}$, so that passing to the limit $\alpha \rightarrow 1$ yields, by Lemma 2.4 and (57), that (62) holds for all $0<\alpha \leqslant 1$ and $p \in\{1, \infty\}$.

Then let us prove (63). Assume that $0<\alpha<1$. Combining (64) and (71) gives that

$$
\left(\mathcal{S}_{\alpha, \tau}\left(v \widehat{\delta}_{0}\right)\right)\left(t_{j}-\right)=\mathcal{E}_{\alpha}\left(t_{j}-\right) v, \quad 1 \leqslant j \leqslant J,
$$

and so (28) implies

$$
\begin{aligned}
& \left\|\mathcal{S}_{\alpha}\left(v \delta_{0}\right)-\mathcal{S}_{\alpha, \tau}\left(v \widehat{\delta}_{0}\right)\right\|_{L^{1}\left(0, T ;[X, Y]_{\theta}\right)} \\
\leqslant & \left\|E_{\alpha}-\mathcal{E}_{\alpha}\right\|_{L^{1}\left(0, T ; \mathcal{L}\left(Y,[X, Y]_{\theta}\right)\right)}\|v\|_{Y} .
\end{aligned}
$$

Therefore, (78) proves that (63) holds for each $0<\alpha<1$. A simple modification of the proof of (37) gives

$$
\lim _{\alpha \rightarrow 1-}\left\|E_{\alpha}-E_{1}\right\|_{L^{1}\left(0, T ; \mathcal{L}\left(Y,[X, Y]_{\theta}\right)\right)}=0,
$$

so that (28) implies

$$
\lim _{\alpha \rightarrow 1-}\left\|\mathcal{S}_{\alpha}\left(v \delta_{0}\right)-\mathcal{S}_{1}\left(v \delta_{0}\right)\right\|_{L^{1}\left(0, T ;[X, Y]_{\theta}\right)}=0 .
$$

Moreover, (57) yields

$$
\lim _{\alpha \rightarrow 1-}\left\|\mathcal{S}_{\alpha, \tau}\left(v \widehat{\delta}_{0}\right)-\mathcal{S}_{1, \tau}\left(v \widehat{\delta}_{0}\right)\right\|_{L^{1}\left(0, T ;[X, Y]_{\theta}\right)}=0 .
$$

Therefore, passing to the limit $\alpha \rightarrow 1-$ in (63) yields that (63) holds with $\alpha=1$. This completes the proof of Lemma 2.7. 


\section{A Dirichlet boundary control problem}

Assume that $0<\alpha \leqslant 1,0<T<\infty$, and $\Omega \subset \mathbb{R}^{d}(d=2,3)$ is a bounded convex polygonal domain with boundary $\partial \Omega$. Define

$U_{\text {ad }}:=\left\{v \in L^{2}\left(0, T ; L^{2}(\partial \Omega)\right): u_{*} \leqslant v(x, t) \leqslant u^{*}\right.$ for a.e. $\left.(x, t) \in \partial \Omega \times(0, T)\right\}$, where $u_{*}$ and $u^{*}$ are two given constants. For any $y \in C\left((0, T] ; L^{2}(\Omega)\right)$ and $u \in L^{2}\left(0, T ; L^{2}(\partial \Omega)\right)$, define

$$
J_{\alpha}(y, u):=\frac{1}{2}\left\|y(T)-y_{d}\right\|_{L^{2}(\Omega)}^{2}+\frac{\nu}{2}\|u\|_{L^{2}\left(0, T ; L^{2}(\partial \Omega)\right)}^{2},
$$

where $y_{d} \in L^{2}(\Omega)$ and $\nu>0$ is a regularization parameter. We are concerned with the following optimal Dirichlet boundary control problem:

$$
\begin{aligned}
& \text { Minimize } J_{\alpha}(y, u) \text { subject to } u \in U_{\text {ad }} \text { and } \\
& \left\{\begin{array}{rlr}
\left(\partial_{0+}^{\alpha}-\Delta\right) y=0 & & \text { in } \Omega \times(0, T) \\
y=u & & \text { on } \partial \Omega \times(0, T) \\
y(\cdot, 0)=0 & & \text { in } \Omega .
\end{array}\right.
\end{aligned}
$$

Here, $\partial_{0+}^{\alpha}$, a fractional partial differential operator, is the scalar-valued version of $\mathrm{D}_{0+}^{\alpha}$ with respect to the time variable $t$.

To apply the theory in the previous section to problem (90), we will use the following settings:

$$
\mathcal{A}:=\Delta ; \quad \mathcal{A}^{*}:=\Delta ; \quad X:=H_{0}^{1}(\Omega) \cap H^{2}(\Omega) ; \quad Y:=L^{2}(\Omega) ; \quad Z:=L^{2}(\partial \Omega) ;
$$

the operator $\mathcal{R}_{\theta_{0}}: Z \rightarrow\left[X^{*}, Y\right]_{\theta_{0}}, 0<\theta_{0}<1 / 4$, is defined by that

$$
\left\langle\mathcal{R}_{\theta_{0}} w, v\right\rangle_{[X, Y]_{\theta_{0}}}:=-\left\langle w, \partial_{\boldsymbol{n}} v\right\rangle_{\partial \Omega}
$$

for all $w \in Z$ and $v \in[X, Y]_{\theta_{0}}$, where $\partial_{\boldsymbol{n}} v$ is the outward normal derivative of $v$ on $\partial \Omega$. By the well-known trace inequality that

$$
\|v\|_{Z} \leqslant \frac{C_{\Omega}}{\sqrt{\epsilon}}\|v\|_{[X, Y]_{3 / 4-\epsilon}} \quad \text { for all } v \in[X, Y]_{3 / 4-\epsilon} \text { with } 0<\epsilon \leqslant 3 / 4,
$$

we readily conclude that, for any $0<\theta_{0}<1 / 4$,

$$
\left\|\mathcal{R}_{\theta_{0}}\right\|_{\mathcal{L}\left(Z,\left[X^{*}, Y\right]_{\theta_{0}}\right)} \leqslant \frac{C_{\Omega}}{\sqrt{1-4 \theta_{0}}} .
$$

Remark 3.1. For the techniques to prove (92), we refer the reader to [56, 3, Ch. VI], [58, Lemmas 16.1 and 23.1] and [42, Corollary 4.37].

Let $u$ and $y$ be defined in Theorem 2.1, and let $U$ and $Y$ be defined in Theorem 2.2. A straightforward calculation gives, by (53) and (93), that

$$
\begin{aligned}
& \|(y-Y)(T-)\|_{Y}+\sqrt{\nu}\|u-U\|_{L^{2}(0, T ; Z)} \\
\leqslant & C_{u_{*}, u^{*}, T, \Omega}\left(\left\|y_{d}\right\|_{Y}+\left(1-4 \theta_{0}\right)^{-1 / 2}\right)\left(\theta_{0} \alpha\right)^{-1} \tau^{\theta_{0} \alpha / 2}
\end{aligned}
$$


for all $0<\theta_{0}<1 / 4$. Assuming $\tau<\exp (-4)$ and inserting $\theta_{0}=1 / 4-1 / \ln (1 / \tau)$ into the above inequality, we then obtain

$$
\begin{aligned}
& \|(y-Y)(T-)\|_{Y}+\sqrt{\nu}\|u-U\|_{L^{2}(0, T ; Z)} \\
\leqslant & C_{u_{*}, u^{*}, T, \Omega} \alpha^{-1}\left(\left\|y_{d}\right\|_{L^{2}(\Omega)}+\sqrt{\ln (1 / \tau)}\right) \tau^{\alpha / 8} .
\end{aligned}
$$

Remark 3.2. For the case $\alpha=1$, [29, 30, 33, 31, 32] used

$$
-\mathcal{A} \int_{0}^{t} E_{1}(t-s)(-\mathcal{A})^{-1} \mathcal{R}_{\theta_{0}} u(s) \mathrm{d} s, \quad 0 \leqslant t \leqslant T,
$$

as the solution of the state equation of problem (90) with $u \in L^{2}(0, T ; Z)$. It is evident that the above solution is exactly $\mathcal{S}_{1} \mathcal{R}_{\theta_{0}} u$.

It remains to prove that $\mathcal{S}_{\alpha} \mathcal{R}_{\theta_{0}} u$ is a sensible solution to the state equation of problem (90) for each $u \in L^{2}(0, T ; Z)$. To this end, we first introduce the very weak solution concept of the state equation, following the idea in [40]. For any $0<\alpha<1$ and $g \in L^{2}(0, T ; Y)$, there exists a unique $w \in{ }^{0} H^{\alpha}(0, T ; Y) \cap$ $L^{2}(0, T ; X)$ such that (cf. $\left.[36,43]\right)$

$$
\left(\mathrm{D}_{T-}^{\alpha}-\mathcal{A}\right) w=g
$$

and

$$
\|w\|_{{ }_{H^{\alpha}(0, T ; Y)}}+\|w\|_{L^{2}(0, T ; X)} \leqslant C_{\alpha}\|g\|_{L^{2}\left(0, T ; L^{2}(\Omega)\right)} .
$$

For $\alpha=1$ the above results are standard (cf. [12]). Hence, by the method of transposition (cf. [40]), we define the very weak solution $y \in L^{2}(0, T ; Y)$ to the state equation of problem (90) with $u \in L^{2}(0, T ; Z)$ by that

$$
\int_{0}^{T}\left(y,\left(\mathrm{D}_{T-}^{\alpha}-\mathcal{A}\right) \varphi\right)_{Y} \mathrm{~d} t=-\left\langle u, \partial_{\boldsymbol{n}} \varphi\right\rangle_{\partial \Omega \times(0, T)}
$$

for all $\varphi \in{ }^{0} H^{\alpha}(0, T ; Y) \cap L^{2}(0, T ; X)$.

Then we will prove that, for any $0<\theta_{0}<1 / 4$ and $u \in L^{2}(0, T ; Z), \mathcal{S}_{\alpha} \mathcal{R}_{\theta_{0}} u$ is identical to the very weak solution to the state equation of problem (90), and hence the application of the theory in the previous section to problem (90) is reasonable.

Lemma 3.1. Assume that $0<\alpha \leqslant 1$ and $0<\theta_{0}<1 / 4$. Then $\mathcal{S}_{\alpha} \mathcal{R}_{\theta_{0}} u$ is the very weak solution to the state equation of problem (90) for each $u \in L^{2}(0, T ; Z)$.

Proof. We only prove the case $0<\alpha<1$, the proof of the case $\alpha=1$ being easier. Assume first that $u \in C([0, T] ; Z)$. By Lemma 2.5 we have

$$
\left(\mathrm{D}_{0+}^{\alpha}-\mathcal{A}\right) \mathcal{S}_{\alpha} \mathcal{R}_{\theta_{0}} u=\mathcal{R}_{\theta_{0}} u
$$

and

$$
\mathrm{D}_{0+}^{\alpha} \mathcal{S}_{\alpha} \mathcal{R}_{\theta_{0}} u, \mathcal{A S}_{\alpha} \mathcal{R}_{\theta_{0}} u \in C\left([0, T] ; X^{*}\right) .
$$

Hence, for any $\varphi \in{ }^{0} H^{\alpha}(0, T ; Y) \cap L^{2}(0, T ; X)$ we have

$$
\int_{0}^{T}\left\langle\left(\mathrm{D}_{0+}^{\alpha}-\mathcal{A}\right) \mathcal{S}_{\alpha} \mathcal{R}_{\theta_{0}} u, \varphi\right\rangle_{X} \mathrm{~d} t=\int_{0}^{T}\left\langle\mathcal{R}_{\theta_{0}} u, \varphi\right\rangle_{X} \mathrm{~d} t .
$$


Because (95) implies $\mathrm{D}_{0+}^{\alpha} \mathcal{S}_{\alpha} \mathcal{R}_{\theta_{0}} u \in L^{2}\left(0, T ; X^{*}\right)$, by [43, Lemma 3.4] we have

$$
\mathcal{S}_{\alpha} \mathcal{R}_{\theta_{0}} u \in{ }_{0} H^{\alpha}\left(0, T ; X^{*}\right) \text {. }
$$

Also, (95) and (15) imply

$$
\mathcal{S}_{\alpha} \mathcal{R}_{\theta_{0}} u \in L^{2}(0, T ; Y)
$$

Consequently, by (4) we have

$$
\int_{0}^{T}\left\langle\mathrm{D}_{0+}^{\alpha} \mathcal{S}_{\alpha} \mathcal{R}_{\theta_{0}} u, \varphi\right\rangle_{X} \mathrm{~d} t=\int_{0}^{T}\left(\mathcal{S}_{\alpha} \mathcal{R}_{\theta_{0}} u, \mathrm{D}_{T-}^{\alpha} \varphi\right)_{Y} \mathrm{~d} t
$$

and it is evident by (6) that

$$
\int_{0}^{T}\left\langle-\mathcal{A S}_{\alpha} \mathcal{R}_{\theta_{0}} u, \varphi\right\rangle_{X} \mathrm{~d} t=\int_{0}^{T}\left(\mathcal{S}_{\alpha} \mathcal{R}_{\theta_{0}} u,-\mathcal{A}^{*} \varphi\right)_{Y} \mathrm{~d} t .
$$

Combining (96) and the above two equations gives

$$
\int_{0}^{T}\left(\mathcal{S}_{\alpha} \mathcal{R}_{\theta_{0}} u,\left(\mathrm{D}_{T-}^{\alpha}-\mathcal{A}^{*}\right) \varphi\right)_{Y} \mathrm{~d} t=\int_{0}^{T}\left\langle\mathcal{R}_{\theta_{0}} u, \varphi\right\rangle_{X} \mathrm{~d} t .
$$

The arbitrariness of $\varphi \in{ }^{0} H^{\alpha}(0, T ; Y) \cap L^{2}(0, T ; X)$ proves that $\mathcal{S}_{\alpha} \mathcal{R}_{\theta_{0}} u$ is indeed the very weak solution. The general case $u \in L^{2}(0, T ; Z)$ then follows from a standard density argument by

$$
\mathcal{S}_{\alpha} \mathcal{R}_{\theta_{0}} \in \mathcal{L}\left(L^{2}(0, T ; Z), L^{2}(0, T ; Y)\right),
$$

which is a direct consequence of $(32)$ and the fact $\mathcal{R}_{\theta_{0}} \in \mathcal{L}\left(Z,\left[X^{*}, Y\right]_{\theta_{0}}\right)$. This completes the proof.

\section{Numerical results}

This section performs three numerical experiments in two-dimensional space to verify the theoretical results. We will use the following settings: $\Omega:=(0,1) \times$ $(0,1) ; T=0.1 ; X, Y, Z, \mathcal{R}_{\theta_{0}}$ and $U_{\text {ad }}$ are defined as in Section 3 .

Experiment 1. Define

$$
\begin{aligned}
g(t) & := \begin{cases}1 & \text { if } 0<t<2 / 3 T \\
3 & \text { if } 2 / 3 T<t<T,\end{cases} \\
v(x, y) & := \begin{cases}y^{-1 / 2} & \text { if }(x, y) \in\{(0, y): 0<y<1\}, \\
0 & \text { if }(x, y) \in \partial \Omega \backslash\{(0, y): 0<y<1\} .\end{cases}
\end{aligned}
$$

To approximate $\mathcal{S}_{\alpha} \mathcal{R}_{\theta_{0}}(g v)$, we use discretization $(46)(0<\alpha<1)$ or $(48)(\alpha=$ 1 ) in time and use the usual $H^{1}(\Omega)$-conforming $P 1$-element method in space. Let $U^{M}$ be the corresponding numerical approximation with time step $\tau=$ $T / 2^{M}$ and spatial mesh size $h=2^{-9}$. Estimates (62) and (93) predict that $\left\|U^{M}-U^{13}\right\|_{L^{\infty}\left(0, T ; L^{2}(\Omega)\right)}$ is close to $O\left(\tau^{0.125}\right)$ for $\alpha=0.5$ and close to $O\left(\tau^{0.25}\right)$ for $\alpha=1$, and this is confirmed by the numerical results in Table 1 . 


\begin{tabular}{cccccc}
\hline & \multicolumn{2}{c}{$\alpha=0.5$} & & \multicolumn{2}{c}{$\alpha=1$} \\
\cline { 2 - 3 } \cline { 5 - 6 }$M$ & $\left\|U^{M}-U^{13}\right\|_{L^{\infty}\left(L^{2}\right)}$ & Order & & $\left\|U^{M}-U^{13}\right\|_{L^{\infty}\left(L^{2}\right)}$ & Order \\
4 & $6.93 \mathrm{e}-1$ & - & & $4.34 \mathrm{e}-1$ & - \\
6 & $6.26 \mathrm{e}-1$ & 0.07 & & $3.23 \mathrm{e}-1$ & 0.21 \\
8 & $5.51 \mathrm{e}-1$ & 0.09 & & $2.24 \mathrm{e}-1$ & 0.26 \\
10 & $4.96 \mathrm{e}-1$ & 0.08 & & $1.62 \mathrm{e}-1$ & 0.24 \\
\hline
\end{tabular}

Table 1: $\|\cdot\|_{L^{\infty}\left(L^{2}\right)}$ means the norm $\|\cdot\|_{L^{\infty}\left(0, T ; L^{2}(\Omega)\right)}$

Experiment 2. Define

$$
v(x, y):=x^{-1 / 2} \quad \text { for all }(x, y) \in \Omega .
$$

To approximate $\mathcal{S}_{\alpha}\left(v \delta_{0}\right)$, we use discretization $(46)(0<\alpha<1)$ or $(48)(\alpha=1)$ in time and use the usual $H^{1}(\Omega)$-conforming $P 1$-element method in space. Let $U^{M}$ be the corresponding numerical approximation with time step $\tau=T / 2^{M}$ and spatial mesh size $h=2^{-9}$. Table 2 illustrates that $\left\|U^{M}-U^{13}\right\|_{L^{1}\left(0, T ; H_{0}^{1}(\Omega)\right)}$ is close to $O\left(\tau^{0.25}\right)$ for $\alpha=0.5$ and close to $O\left(\tau^{0.5}\right)$ for $\alpha=1$, which agrees well with estimate (63).

\begin{tabular}{cccccc}
\hline & \multicolumn{2}{c}{$\alpha=0.5$} & & \multicolumn{2}{c}{$\alpha=1$} \\
\cline { 2 - 3 } \cline { 5 - 6 }$M$ & $\left\|U^{M}-U^{13}\right\|_{L^{1}\left(H_{0}^{1}\right)}$ & Order & & $U^{M}-U^{13} \|_{L^{1}\left(H_{0}^{1}\right)}$ & Order \\
4 & $1.71 \mathrm{e}-0$ & - & & $4.24 \mathrm{e}-1$ & - \\
5 & $1.46 \mathrm{e}-0$ & 0.23 & & $3.01 \mathrm{e}-1$ & 0.49 \\
6 & $1.23 \mathrm{e}-0$ & 0.25 & & $2.10 \mathrm{e}-1$ & 0.52 \\
7 & $1.02 \mathrm{e}-0$ & 0.27 & & $1.45 \mathrm{e}-1$ & 0.54 \\
\hline
\end{tabular}

Table 2: $\|\cdot\|_{L^{1}\left(H_{0}^{1}\right)}$ means the norm $\|\cdot\|_{L^{1}\left(0, T ; H_{0}^{1}(\Omega)\right)}$

Experiment 3. Let $\nu:=10, u_{*}:=0, u^{*}:=20$ and

$$
y_{d}(x, y):=1 \text { for all }(x, y) \in \Omega \text {. }
$$

To approximate problem (90), we will use the temporal discretization in Section 2.3 and the $H^{1}(\Omega)$-conforming $P$ 1-element method to discretize the state equation in time and space, respectively; see [16] for the implementation details. Let $U^{M}$ be the corresponding numerical solution with time step $\tau=T / 2^{M}$ and spatial mesh size $h=2^{-8}$. The numerical results in Table 3 show that $\left\|U^{M}-U^{13}\right\|_{L^{2}\left(0, T ; L^{2}(\partial \Omega)\right)}$ is close to $O\left(\tau^{0.125}\right)$, which agrees with error estimate (94).

\begin{tabular}{ccc}
\hline$M$ & $\left\|U^{M}-U^{12}\right\|_{L^{2}\left(0, T ; L^{2}(\partial \Omega)\right)}$ & Order \\
\hline 4 & $2.44 \mathrm{e}-1$ & - \\
5 & $2.08 \mathrm{e}-1$ & 0.23 \\
6 & $1.88 \mathrm{e}-1$ & 0.15 \\
7 & $1.66 \mathrm{e}-1$ & 0.18 \\
\hline
\end{tabular}

Table 3: Numerical results for Experiment 3 with $\alpha=1$ 


\section{References}

[1] W. Alt and U. Mackenroth. Convergence of finite element approximations to state constrained convex parabolic boundary control problems. SIAM J. Control Optim., 27(4):718-736, 1989.

[2] H. Antil, E. Otrola, and A. J. Salgado. A space-time fractional optimal control problem: Analysis and discretization. SIAM J. Control Optim., 54(3):1295-1328, 2016.

[3] A. V. Balakrishnan. Applied functional analysis. Springer, New York, 1981.

[4] F. B. Belgacem, C. Bernardi, and H. E. Fekih. Dirichlet boundary control for a parabolic equation with a final observation I: a space-time mixed formulation and penalization. Asymptot Anal., 71:101-121, 2011.

[5] M. Berggren. Approximations of very weak solutions to boundary-value problems. SIAM J. Numer. Anal., 42(2):860-877, 2004.

[6] A. Caldern. Intermediate spaces and interpolation, the complex method. Stud. Math., 24(2):113-190, 1964.

[7] K. Chrysafinos and E. N. Karatzas. Error estimates for discontinuous Galerkin time-stepping schemes for robin boundary control problems constrained to parabolic pdes. SIAM J. Numer. Anal., 52(6):2837-2862, 2014.

[8] K. Deckelnick and M. Hinze. Variational discretization of parabolic control problems in the presence of pointwise state constraints. J. Comput. Math., $29: 1-15,2011$.

[9] K. Diethelm. The analysis of fractional differential equations. Springer, Berlin, 2010.

[10] K. Eriksson, C. Johnson, and V. Thomée. Time discretization of parabolic problems by the discontinuous Galerkin method. RAIRO Modél. Math. Anal. Numér., 19:611-643, 1985.

[11] V. Ervin and J. Roop. Variational formulation for the stationary fractional advection dispersion equation. Numer. Meth. Part. D. E., 22(3):558-576, 2006.

[12] L. C. Evans. Partial differential equations. American Mathematical Society, 2 edition, 2010.

[13] D. A. French and J. T. King. Approximation of an elliptic control problem by the finite element method. Numer. Funct. Anal. Optim., 12:299-314, 1991.

[14] D. A. French and J. T. King. Analysis of a robust finite element approximation for a parabolic equation with rough boundary data. Math. Comput., 60:79-104, 1993.

[15] W. Gong, M. Hinze, and Z. Zhou. A priori error analysis for finite element approximation of parabolic optimal control problems with pointwise control. SIAM J. Control Optim., 52:97-119, 2014. 
[16] W. Gong, M. Hinze, and Z. Zhou. Finite element method and a priori error estimates for Dirichlet boundary control problems governed by parabolic pdes. J. Sci. Comput., 66:941-967, 2016.

[17] W. Gong and B. Li. Improved error estimates for semidiscrete finite element solutions of parabolic Dirichlet boundary control problems. IMA J. Numer. Anal., 0:1-42, 2019.

[18] M. Gunzburger and J. Wang. Error analysis of fully discrete finite element approximations to an optimal control problem governed by a time-fractional pde. SIAM J. Control Optim., 57(1):241-263, 2019.

[19] M. Hinze. A variational discretization concept in control constrained optimization: the linear-quadratic case. Comput. Optim. Appl., 30:45-63, 2005.

[20] B. Jin, R. Lazarov, J. Pasciak, and Z. Zhou. Error analysis of semidiscrete finite element methods for inhomogeneous time-fractional diffusion. IMA J. Numer. Anal., 35:561-582, 2015.

[21] B. Jin, R. Lazarov, and Z. Zhou. Error estimates for a semidiscrete finite element method for fractional order parabolic equations. SIAM J. Numer. Anal., 51(1):445-466, 2013.

[22] B. Jin, R. Lazarov, and Z. Zhou. An analysis of the L1 scheme for the subdiffusion equation with nonsmooth data. IMA J. Numer. Anal., 36:197$221,2016$.

[23] B. Jin, R. Lazarov, and Z. Zhou. Two fully discrete schemes for fractional diffusion and diffusion-wave equations with nonsmooth data. SIAM J. Sci. Comput., 38(1):A146-A170, 2016.

[24] B. Jin, B. Li, and Z. Zhou. Discrete maximal regularity of time-stepping schemes for fractional evolution equations. Numer. Math., 138:101-131, 2018.

[25] B. Jin, B. Li, and Z. Zhou. Subdiffusion with a time-dependent coefficient: analysis and numerical solution. Math. Comp., 88:2157-2186, 2019.

[26] B. Jin, B. Li, and Z. Zhou. Pointwise-in-time error estimates for an optimal control problem with subdiffusioin constraint. IMA J. Numer. Anal., 40:377-404, 2020.

[27] G. Knowles. Finite element approximation of parabolic time optimal control problems. SIAM J. Control Optim., 20(3):414-427, 1982.

[28] K. Kunisch and B. Vexler. Constrained Dirichlet boundary control in $L^{2}$ for a class of evolution equations. SIAM J. Control Optim., 46:1726-1753, 2007.

[29] I. Lasiecka. Boundary control of parabolic systems: Finite-element approximation. Appl. Math. Optim., 31:31-62, 1980.

[30] I. Lasiecka. Unified theory for abstract parabolic boundary problemsa semigroup approach. Appl. Math. Optim., 6:287-333, 1980. 
[31] I. Lasiecka. RitzGalerkin approximation of the time optimal boundary control problem for parabolic systems with Dirichlet boundary conditions. SIAM J. Control Optim., 22(3):477-500, 1984.

[32] I. Lasiecka. Galerkin approximations of abstract parabolic boundary value problems with rough boundary data- $L_{p}$ theory. Math. Comput., 47:55-75, 1986.

[33] I. Lasiecka and R. Triggiani. Dirichlet boundary control problem for parabolic equations with quadratic cost: analyticity and Riccatis feedback synthesis. SIAM J. Control Optim., 21(1):41-67, 1983.

[34] D. Leykekhman and B. Vexler. Optimal a priori error estimates of parabolic optimal problems with pointwise control. SIAM J. Numer. Anal., 51:2797$2821,2013$.

[35] D. Leykekhman and B. Vexler. A priori error estimates for three dimensional parabolic optimal control problems with pointwise control. SIAM J. Numer. Anal., 54:2403-2435, 2016.

[36] B. Li, H. Luo, and X. Xie. Analysis of a time-stepping scheme for time fractional diffusion problems with nonsmooth data. SIAM J. Numer. Anal., 57(2):779-798, 2019.

[37] B. Li, T. Wang, and X. Xie. Numerical analysis of two Galerkin discretizations with graded temporal grids for fractional evolution equations. submitted, axXiv:2002.11914, 2020.

[38] B. Li, X. Xie, and Y. Yan. L1 scheme for solving an inverse problem subject to a fractional diffusion equation. submitted, arXiv:2006.04291, 2020.

[39] Y Lin and C. Xu. Finite difference/spectral approximations for the timefractional diffusion equation. J. Comput. Phys., 225(2):1533-1552, 2007.

[40] J. L. Lions and E. Magenes. Non-homogeneous boundary value problems and applications. Springer, Berlin, 1972.

[41] C. Lubich, I. Sloan, and V. Thomée. Nonsmooth data error estimates for approximations of an evolution equation with a positive-type memory term. Math. Comput., 65(213):1-17, 1996.

[42] A. Lunardi. Interpolation theory. Edizioni della Normale, Pisa, 2018.

[43] H. Luo, B. Li, and X. Xie. Convergence analysis of a Petrov-Galerkin method for fractional wave problems with nonsmooth data. J. Sci. Comput., 80(2):957-992, 2019.

[44] K. Malanowski. Convergence of approximations vs. regularity of solutions for convex, control-constrained optimal-control problems. Appl. Math. Optim., 8:69-95, 1981.

[45] W. Mclean and K. Mustapha. Convergence analysis of a discontinuous Galerkin method for a sub-diffusion equation. Numer. Algor., 52(1):69-88, 2009. 
[46] W. McLean and V. Thomée. Numerical solution via Laplace transforms of a fractional order evolution equation. J. Integral Equ. Appl., 22:57-94, 03 2010 .

[47] D. Meidner and B. Vexler. Adaptive space-time finite element methods for parabolic optimization problems. SIAM J. Control Optim., 46:116-142, 2007.

[48] D. Meidner and B. Vexler. A priori error estimates for space-time finite element discretization of parabolic optimal control problems part I: problems without control constraints. SIAM J. Control Optim., 47:1150-1177, 2008.

[49] D. Meidner and B. Vexler. A priori error estimates for space-time finite element discretization of parabolic optimal control problems part II: Problems with control constraints. SIAM J. Control Optim., 47(3):1301-1329, 2008.

[50] D. Meidner and B. Vexler. A priori error analysis of the Petrov-Galerkin Crank-Nicolson scheme for parabolic optimal control problems. SIAM J. Control Optim., 49(5):2183-2211, 2011.

[51] K. Mustapha, B. Abdallah, and K. Furati. A discontinuous Petrov-Galerkin method for time-fractional diffusion equations. Fuel, 58(12):896-897, 2014.

[52] K. Mustapha and W. Mclean. Discontinuous Galerkin method for an evolution equation with a memory term of positive type. Math. Comput., 78(268):1975-1995, 2009.

[53] K. Mustapha and W. McLean. Piecewise-linear, discontinuous Galerkin method for a fractional diffusion equation. Numer. Algor., 56(2):159-184, 2011.

[54] A. Pazy. Semigroups of linear operators and applications to partial differential equations. Springer, Berlin, 1983.

[55] I. Podlubny. Fractional differential equations. Academic Press, 1998.

[56] E. M. Stein. Singular integrals and differentiability properties of functions. Princeton University Press, Princeton, 1970.

[57] Z. Sun and X. Wu. A fully discrete difference scheme for a diffusion-wave system. Appl. Numer. Math., 56(2):193-209, 2006.

[58] L. Tartar. An introduction to Sobolev spaces and interpolation spaces. Springer, Berlin, 2007.

[59] V. Thomée. Galerkin finite element methods for parabolic problems. Springer, Berlin, 2006.

[60] F. Troltzsch. Optimal control of partial differential equations. American Mathematical Society, 2010.

[61] C. Zhang, H. Liu and Z. Zhou. A priori error analysis for time-stepping discontinuous Galerkin finite element approximation of time fractional optimal control problem. J. Sci. Comput., 80:993-1018, 2019. 